\title{
ON UNIQUENESS OF TENSOR PRODUCTS OF IRREDUCIBLE CATEGORIFICATIONS
}

\author{
IVAN LOSEV AND BEN WEBSTER
}

\begin{abstract}
In this paper, we propose an axiomatic definition for a tensor product categorification. A tensor product categorification is an abelian category with a categorical action of a Kac-Moody algebra $\mathfrak{g}$ in the sense of Rouquier or Khovanov-Lauda whose Grothendieck group is isomorphic to a tensor product of simple modules. However, we require a much stronger structure than a mere isomorphism of representations; most importantly, each such categorical representation must have a standardly stratified structure compatible with the categorification functors, and with combinatorics matching those of the tensor product.

With these stronger conditions, we recover a uniqueness theorem similar in flavor to that of Rouquier for categorifications of simple modules. Furthermore, we already know of an example of such a categorification: the representation category of an algebra $T^{\boldsymbol{\lambda}}$ previously defined by the second author using generators and relations. Next, we show that tensor product categorifications give a categorical realization of tensor product crystals analogous to that for simple crystals given by cyclotomic quotients of KLR algebras.

Examples of such categories are also readily found in more classical representation theory; for finite and affine type A, tensor product categorifications can be realized as quotients of the representation categories of cyclotomic $q$-Schur algebras.
\end{abstract}

\section{INTRODUCTION}

A subject that has attracted great attention in recent years is that of categorical representations of a Kac-Moody Lie algebra $\mathfrak{g}$; this is the study of 2-categories corresponding to universal enveloping algebras of Lie algebras, and in particular, their actions on categories. This theory has deep roots, but the notion of a categorical action of $\mathfrak{s l}_{2}$ was first introduced by Chuang and Rouquier CR08, and broadened to other Kac-Moody algebras and developed further by Khovanov and Lauda [Lau10, KL10] and Rouquier [Rou].

Obviously, one important question is the relationship between categorical representations and the usual linear representations of $\mathfrak{g}$. For simple representations, this relationship is quite direct: each simple linear representation of $\mathfrak{g}$ has a universal categorification, by work of Rouquier [Rou]. In essence, simple linear representations and simple categorical representations are in bijection.

However, categorical representations are not necessarily semi-simple, even if the representation on their Grothendieck group is. Examples show that interesting non-irreducible representations should have categorifications which are "more than the sum of their parts"; in this paper, our main example is tensor products of irreducibles, but the same principle applies to the categorifications of Fock space supplied by the categories $\mathcal{O}$ over Cherednik algebras Sha11].

In particular, the second author defined a category $\mathfrak{V}^{\underline{\nu}}$ attached to a list of highest weights $\underline{\boldsymbol{\nu}}=\left(\nu_{1}, \ldots, \nu_{\ell}\right)$ in [Weba]; in this paper, we will denote this category $\mathcal{C}(\underline{\boldsymbol{\nu}})$. This carries a

MSC 2010: 16G99, 17B10, 18D99. 
categorical action of $\mathfrak{g}$ and has Grothendieck group isomorphic to the tensor product of $U(\mathfrak{g})$ modules $V_{\nu_{1}} \otimes \cdots \otimes V_{\nu_{\ell}}$ (or the corresponding representations of $U_{q}(\mathfrak{g})$ if one incorporates the grading). Many properties of this category suggest that it is the "right" categorification for tensor products; in particular, the ribbon structure on the category of $U_{q}(\mathfrak{g})$ modules, and the canonical basis of Lusztig both have appropriate categorical analogues. However, the definition given in [Weba] is ad hoc, defined by generators and relations, and lacks a universal property. In this paper, we try to correct this defect, giving an axiomatic characterization of this category, suggested by the notion of a highest weight categorification introduced by the first author [Losa, Losb].

In the crudest sense, the category $\mathcal{C}(\underline{\boldsymbol{\nu}})$ is the unique abelian category which carries a categorical $\mathfrak{g}$-action and which is obtained by beginning with the unique categorification of $V_{\nu_{1}} \otimes \cdots \otimes V_{\nu_{\ell}}$ as an irreducible $\mathfrak{g}^{\oplus \ell}$-module, and then adding in new extensions between the projectives in this category in a controlled way. We require that the resulting category is standardly stratified, that is, it has a subcategory of standardly filtered modules which is closed under categorification functors, and whose combinatorics are controlled by those of the tensor product (in particular, the preorder used in the stratification depends on the order of the tensor factors). We formalize this idea with the definition of a tensor product categorification ( $(3.2)$. The main body of the paper is dedicated to the proof of the uniqueness result described above:

Theorem A (Thm. 6.1, Thm. 17.2). Any tensor product categorification for the representation $V_{\nu_{1}} \otimes \cdots \otimes V_{\nu_{\ell}}$ is strongly equivariantly equivalent to $\mathcal{C}(\underline{\boldsymbol{\nu}})$ as a categorical $\mathfrak{g}$-module.

For any such categorification, there is a canonical isomorphism of crystals between the isomorphism classes of simple objects and the tensor product crystal $B\left(\nu_{1}\right) \otimes \cdots \otimes B\left(\nu_{\ell}\right)$ (as conjectured in [Weba, 3.12]).

The proof is by induction; we use the notion of categorical splitting, introduced by the first author in [Losb]. Roughly, inside any categorification of $V_{\nu_{1}} \otimes \cdots \otimes V_{\nu_{\ell}}$, one finds a categorification of $V_{\nu_{1}} \otimes \cdots \otimes V_{\nu_{\ell-1}}$. The inductive hypothesis allows us to identify this subcategory with $\mathcal{C}\left(\nu_{1}, \ldots, \nu_{\ell-1}\right)$; we can then argue that any tensor product $\mathfrak{g}$-categorification containing an appropriately embedded copy of $\mathcal{C}\left(\nu_{1}, \ldots, \nu_{\ell-1}\right)$ must be $\mathcal{C}(\underline{\boldsymbol{\nu}})$.

This theorem has quite powerful applications in the theory of Lie superalgebras, which will be explored further is a forthcoming joint paper of Brundan and the authors [BLW].

We should note that this theorem is likely not the last word in the question of how one can categorify a tensor product. Of particular import is work announced by Rouquier, which proposes a notion of internal tensor product for the 2-category of categorical $\mathfrak{g}$-actions. Obviously, we anticipate that our tensor product categorifications are the categories we would arrive at using Rouquier's internal tensor product, but this remains to be confirmed.

\section{ACKNOWLEDGEMENTS}

We would like to thank Jon Brundan for his remarks on the previous version of this paper.

I.L. was supported by the NSF under Grant DMS-1161584. B.W. was supported by the NSF under Grant DMS-1151473.

\section{Standardly STRATIFIED CATEGORIES}

2.1. Definition. Let $\mathbb{K}$ be a field of any characteristic. We consider an abelian category $\mathcal{C}$ such that each block of $\mathcal{C}$ is equivalent to the category of finite dimensional modules over a finite dimensional $\mathbb{K}$-algebra, and such that for every simple $\operatorname{End}_{\mathcal{C}}(L) \cong \mathbb{K}$, i.e. every 
irreducible in $\mathcal{C}$ is absolutely irreducible. We will consider a set $\Lambda$ with fixed bijection to the isomorphism classes of simple objects in $\mathcal{C}$. For any $\lambda \in \Lambda$, we let $L(\lambda)$ denote the corresponding simple and let $P(\lambda)$ be its projective cover.

Now consider a poset $\Xi$ with the sets $\left\{\xi^{\prime} \in \Xi \mid \xi^{\prime}>\xi\right\}$ and $\left\{\xi^{\prime} \in \Xi \mid \xi^{\prime}<\xi\right\}$ finite for each $\xi \in \Xi$. Choose a map $\varrho: \Lambda \rightarrow \Xi$ with finite fibers; this map induces a natural preorder on $\Lambda$. To each $\xi \in \Xi$, we assign the Serre subcategories $\mathcal{C}_{\leqslant \xi}\left(\right.$ resp., $\mathcal{C}_{<\xi}$ ) of $\mathcal{C}$ spanned by $L(\lambda)$ with $\varrho(\lambda) \leqslant \xi$ (resp., $\varrho(\lambda)<\xi$ ). Of course, if $\xi \leqslant \xi^{\prime}$, then $\mathcal{C}_{\xi} \subset \mathcal{C}_{\xi^{\prime}}$. For $\xi \in \Xi$, set $\mathcal{C}_{\xi}:=\mathcal{C}_{\leqslant \xi} / \mathcal{C}_{<\xi}$. For $\lambda \in \varrho^{-1}(\xi)$ let $L_{\xi}(\lambda)$ denote the simple object in $\mathcal{C}_{\xi}$ corresponding to $\lambda$. Let $P_{\xi}(\lambda)$ denote the projective cover of $L_{\xi}(\lambda)$ in $\mathcal{C}_{\xi}$.

Let $\pi_{\xi}$ denote the quotient functor $\mathcal{C}_{\leqslant \xi} \rightarrow \mathcal{C}_{\xi}$. We suppose that this functor has an exact left adjoint functor.

Definition 2.1. We will call this left adjoint the standardization functor and denote it by $\Delta_{\xi}$. We will often omit $\xi$ from the notation. For $\lambda \in \Lambda$ let $\Delta(\lambda)$ (resp., $\bar{\Delta}(\lambda)$ ) denote the object $\Delta_{\varrho(\lambda)}\left(P_{\xi}(\lambda)\right)$ (resp., $\Delta_{\varrho(\lambda)}\left(L_{\xi}(\lambda)\right)$ ). The objects $\Delta(\lambda), \bar{\Delta}(\lambda)$ will be called standard and proper standard.

We call the category $\mathcal{C}$ equipped with a filtration $\mathcal{C}_{\leqslant \xi}$ (such that $\Delta_{\xi}$ is an exact functor) a standardly stratified category if there is an epimorphism $P(\lambda) \rightarrow \Delta(\lambda)$ whose kernel admits a filtration by objects $\Delta(\mu)$ with $\mu>\lambda$.

If, for each $\xi, \mathcal{C}_{\xi}$ coincides with the category Vect of vector spaces, then we arrive at the usual definition of a highest weight category.

We remark that, by the definition of $\Delta(\lambda), \bar{\Delta}(\lambda)$, there is an epimorphism $\Delta(\lambda) \rightarrow \bar{\Delta}(\lambda)$ and the head of $\Delta(\lambda)$ is simple and coincides with $L(\lambda)$. We also remark that the simple constituents of the radical of $\bar{\Delta}(\lambda)$ are of the form $L(\mu)$ with $\mu<\lambda$.

It is a standard fact that the condition on a filtration of projectives implies

$$
\begin{aligned}
& \operatorname{Ext}_{\mathcal{C}}^{i}\left(\Delta_{\xi}(M), \Delta_{\xi^{\prime}}\left(M^{\prime}\right)\right)=0, \text { for } \xi \not \xi^{\prime}, i>0 . \\
& \operatorname{Ext}_{\mathcal{C}}^{i}\left(\Delta_{\xi}(M), \Delta_{\xi}\left(M^{\prime}\right)\right)=\operatorname{Ext}_{\mathcal{C}_{\xi}}^{i}\left(M, M^{\prime}\right), i \geqslant 0 .
\end{aligned}
$$

Let $\mathcal{C}^{\Delta}$ (resp., $\mathcal{C}^{\bar{\Delta}}$ ) denote the full subcategory of $\mathcal{C}$ consisting of all objects admitting a filtration whose successive quotients are standard (resp., proper standard) objects. So, in particular, $\mathcal{C}-$ proj $\subset \mathcal{C}^{\Delta} \subset \mathcal{C}^{\bar{\Delta}}$. The following lemma is a direct corollary of (2.1).

Lemma 2.2. Let $\iota_{\xi}$ be the inclusion functor $\mathcal{C}_{\leqslant \xi} \hookrightarrow \mathcal{C}$ and $\iota_{\xi}$ be its left adjoint functor $\mathcal{C} \rightarrow \mathcal{C}_{\leqslant \xi}$. Then the functor $\iota_{\xi}^{!}$is exact on $\mathcal{C}^{\bar{\Delta}}$ (meaning that it maps exact sequences to exact sequences).

For any standardly stratified category $\mathcal{C}$, we can consider its associated graded $\operatorname{gr} \mathcal{C}=\oplus_{\xi} \mathcal{C}_{\xi}$; we can view $\Delta:=\bigoplus_{\xi \in \Xi} \Delta_{\xi}$ as a faithful inclusion gr $\mathcal{C} \rightarrow \mathcal{C}$, which fails to be full.

For an ideal $\Xi_{0}$ in $\Xi$ (i.e., $\xi \in \Xi_{0}, \xi^{\prime}<\xi$ implies $\xi^{\prime} \in \Xi_{0}$ ), we can consider the Serre subcategory $\mathcal{C}_{\Xi_{0}} \subset \mathcal{C}$ spanned by $L(\lambda)$ with $\varrho(\lambda) \in \Xi_{0}$ and the quotient $\mathcal{C} / \mathcal{C}_{\Xi_{0}}$. Both these categories have a natural standardly stratified structure.

The quotient functor $\pi_{\Xi_{0}}: \mathcal{C} \rightarrow \mathcal{C} / \mathcal{C}_{\Xi_{0}}$ has a left adjoint $\pi_{\Xi_{0}}^{!}$which is exact on $\left(\mathcal{C} / \mathcal{C}_{\Xi_{0}}\right)^{\bar{\Delta}}$. For each $\xi \in \Xi \backslash \Xi_{0}$, this functor satisfies $\Delta_{\xi}=\pi_{\Xi_{0}}^{!} \circ \Delta_{0, \xi}$, where $\Delta_{0, \xi}$ is the standardization functor for $\mathcal{C} / \mathcal{C}_{\Xi_{0}}$. This is an easy corollary of the triangularity property for the projectives. In particular,

$$
\pi_{\Xi_{0}}^{!}\left(\Delta_{0}(\lambda)\right)=\Delta(\lambda) \quad \pi_{\Xi_{0}}^{!}\left(\bar{\Delta}_{0}(\lambda)\right)=\bar{\Delta}(\lambda)
$$


2.2. Costandard objects. The category $\mathcal{C}^{\text {opp }}$ has a "dual" standardly stratified structure, which we describe here. Let $I(\lambda)$ denote the injective hull of $L(\lambda)$.

Definition 2.3. Define the costandard object $\nabla(\lambda)$ as the maximal subobject of $I(\lambda)$ whose simple constituents are of the form $L(\mu)$ with $\mu \leqslant \lambda$. Also define the proper costandard object $\bar{\nabla}(\lambda)$ as the maximal subobject of $I(\lambda)$ such that the simple subquotients of $\bar{\nabla}(\lambda) / L(\lambda)$ are of the form $L(\mu)$ with $\mu<\lambda$.

Let $\mathcal{C}^{\nabla}$ (resp., $\mathcal{C}^{\bar{\nabla}}$ ) denote the full subcategories of $\mathcal{C}$ consisting of all objects admitting a filtration whose successive quotients are costandard (resp., proper costandard) objects.

\section{Lemma 2.4.}

(1) We have $\operatorname{dim} \operatorname{Ext}^{i}(\Delta(\lambda), \bar{\nabla}(\mu))=\operatorname{dim} \operatorname{Ext}^{i}(\bar{\Delta}(\lambda), \nabla(\mu))=\delta_{i, 0} \delta_{\lambda, \mu}$. Moreover, $\nabla(\lambda)$ is injective in $\mathcal{C}_{\leqslant \varrho(\lambda)}$.

(2) For $N \in \mathcal{C}$, we have $N \in \mathcal{C}^{\nabla}$ (resp., $N \in \mathcal{C}^{\bar{\nabla}}$ ) if and only if $\operatorname{Ext}^{1}(\bar{\Delta}(\lambda), N)=0$ (resp., $\operatorname{Ext}^{1}(\Delta(\lambda), N)=0$ ) for all $\lambda$.

(3) The right adjoint functor $\nabla_{\xi}$ of the projection $\mathcal{C}_{\leqslant \xi} \rightarrow \mathcal{C}_{\xi}$ is exact and

$$
\nabla_{\xi}\left(I_{\xi}(\lambda)\right)=\nabla(\lambda) \quad \nabla_{\xi}\left(L_{\xi}(\lambda)\right)=\bar{\nabla}(\lambda) .
$$

Here we write $I_{\xi}(\lambda)$ for the injective envelope of $L_{\xi}(\lambda)$ in $\mathcal{C}_{\xi}$. We remark that here it is essential that we require the standardization functor to be exact, see [CPS96] for a counterexample.

Proof. Part (1) is a standard calculation using the filtrations on projective and injective modules. Thus we turn to part (2); furthermore, since the proofs are parallel, we only check that $\operatorname{Ext}^{1}(\bar{\Delta}(\lambda), N)=0$ if and only if $N \in \mathcal{C}^{\nabla}$. By induction, we may assume that $\mathcal{C}=\mathcal{C}_{\leqslant \xi}$ and that for objects in $\mathcal{C}_{<\xi}$, our claims are proved.

Let $N_{0}$ be the largest subobject in $N$ belonging to $\mathcal{C}_{<\xi}$. Then for any $\lambda$ with $\varrho(\lambda)<\xi$ we have $\operatorname{Ext}^{1}\left(\bar{\Delta}(\lambda), N_{0}\right) \cong \operatorname{Hom}\left(\bar{\Delta}(\lambda), N / N_{0}\right)=0$. Therefore, by the inductive assumption, $N_{0}$ is $\nabla$-filtered and hence $\operatorname{Ext}^{i}\left(\bar{\Delta}(\lambda), N_{0}\right)=0$ for all $i>0$. It follows that $\operatorname{Ext}^{1}\left(\bar{\Delta}(\lambda), N / N_{0}\right) \cong$ $\operatorname{Ext}^{2}\left(\bar{\Delta}(\lambda), N_{0}\right)=0$. So it is enough to consider the case when $N_{0}=0$, i.e., the socle of $N$ is a sum of simples of the form $L(\mu)$ for $\varrho(\mu)=\xi$. It follows that $N$ embeds to the sum $I$ of several $I(\mu)$ 's with $\varrho(\mu)=\xi$ such that the socles of $I$ and of $N$ coincide. But for such $\mu$ we have $I(\mu)=\nabla(\mu)$. If we have $\operatorname{Ext}^{1}(\bar{\Delta}(\lambda), N)=0$ for all $\lambda$, then we have a surjection $\operatorname{Hom}(\bar{\Delta}(\lambda), I) \rightarrow \operatorname{Hom}(\bar{\Delta}(\lambda), I / N)$. But since $I$ is the sum of costandard objects, all homomorphisms from $\bar{\Delta}(\lambda)$ to $I$ factor through the socle of $I$ and hence through $N$. So $\operatorname{Hom}(\bar{\Delta}(\lambda), I / N)=0$ for all $\lambda$ and therefore $I / N=0$. Thus, $N=I$ has a costandard filtration.

Part (3) follows immediately; consider an exact sequence $0 \rightarrow E_{1} \rightarrow E \rightarrow E_{2} \rightarrow 0$ in $\mathcal{C}_{\xi}$. We can assume by induction that both $\nabla\left(E_{1}\right), \nabla\left(E_{2}\right)$ are $\bar{\nabla}$-filtered. The cokernel $N$ of $\nabla_{\xi}\left(E_{1}\right) \hookrightarrow \nabla_{\xi}(E)$ satisfies $\operatorname{Ext}^{1}(\Delta(\lambda), N)=0$ for all $\lambda$ and so is $\bar{\nabla}$-filtered. So we have an embedding $N \hookrightarrow \nabla_{\xi}\left(E_{2}\right)$ of $\bar{\nabla}$-filtered objects that becomes an isomorphism after projecting to $\mathcal{C}_{\xi}$. This forces the costandard quotients of $N, \nabla_{\xi}\left(E_{2}\right)$ to be the same and the embedding to be an isomorphism (recall that all blocks of $\mathcal{C}$ are finite).

We also point out the following form of the BGG reciprocity.

Lemma 2.5. The multiplicity $[P(\lambda): \Delta(\mu)]$ of $\Delta(\mu)$ in $P(\lambda)$ equals to the multiplicity $[\bar{\nabla}(\mu): L(\lambda)]$. Similarly, the multiplicity $[I(\lambda): \nabla(\mu)]$ equals $[\bar{\Delta}(\mu): L(\lambda)]$.

Combining these results, we see that: 
Proposition 2.6. The category $\mathcal{C}^{o p p}$ is standardly stratified with respect to the map $\varrho: \Lambda \rightarrow$ $\Xi$ and the standardization functor $\nabla_{\xi}: \mathcal{C}_{\xi}^{o p p} \rightarrow \mathcal{C}_{\leqslant \xi}^{o p p}$.

Remark 2.7. For highest weight categories, one can define tilting objects and has the Ringel duality. This can be generalized to standardly stratified categories. Namely, we say that an object $M$ in a standardly stratified category $\mathcal{C}$ is tilting if it is both $\Delta$-filtered and $\bar{\nabla}$-filtered (there is also a dual notion of a co-tilting object that has to be $\bar{\Delta}$ - and $\nabla$-filtered). Then, similarly to the highest weight case, one can show that every standard object $\Delta(\lambda)$ has a unique tilting hull $T(\lambda)$; the $T(\lambda)$ 's are pairwise non-isomorphic and exhaust all indecomposable tiltings. Set $T:=\bigoplus_{\lambda \in \Lambda} T(\lambda)$. By the Ringel dual $\mathcal{C}^{\vee}$ of $\mathcal{C}$ we mean the category of finite dimensional right End $(T)$-modules. This category admits a natural standardly stratified structure with standardization functor $\operatorname{Hom}_{\mathcal{C}}(T, \nabla(\bullet))$. One can check the axioms similarly, for example, to [Rou08, 4.1.5]. We have a natural equivalence $\left(\mathcal{C}^{\vee}\right)^{\bar{\Delta}} \cong \mathcal{C}^{\bar{\nabla}}$

\section{TEnsor PRODUCT CATEGORIFICATIONS}

3.1. Categorical $\mathfrak{g}$-actions. Let $\mathfrak{g}$ be a Kac-Moody algebra with its set $\left\{\alpha_{i}, i \in I\right\}$ of simple roots. There are a variety of notions of categorical $\mathfrak{g}$-actions which have appeared in the literature, in the work of Rouquier [Rou, Khovanov and Lauda KL10, Cautis and Lauda [CL and others. Of course, as with all definitions where there is some flexibility, one endeavors to use the weakest version possible when proving facts about objects satisfying said definition and the strongest when showing that an object does satisfy it (though one is often forced to do the opposite).

All of these definitions employ the KLR algebra or quiver Hecke algebra $R$, a sum of finitely generated algebras $R_{\mu}$ attached to every element $\mu$ in the positive cone of the root lattice of $\mathfrak{g}$; we let $R_{k}$ for an integer $k$ denote the sum of the $R_{\mu}$ 's for $\mu$ a sum of $k$ simple roots. We should note that our definition of KLR algebra follows that of Rouquier [Rou, §3.2], and thus involves a choice of polynomials $Q_{i j}(u, v)$ for each pair of elements in the Dynkin diagram with degree in $u$ bounded above by the entry $-a_{i j}$ of the negated Cartan matrix, and similarly the degree in $v$ bounded above by $-a_{j i}$. We say this choice is homogeneous if the polynomial $Q_{i j}(u, v)$ is homogeneous when the ratio between the degrees of the variables $u$ and $v$ equals the ratio between the lengths of the simply roots $\alpha_{i}$ and $\alpha_{j}$. We'll follow the conventions of [Weba, $\S 1$ ] throughout.

The finitely generated modules over the KLR algebra form a monoidal category under induction functors; this monoidal category on its own is a categorification, in a certain sense, of the enveloping algebra $U\left(\mathfrak{g}_{+}\right)$of the Borel $\mathfrak{g}_{+}$.

For our purposes, a categorical $\mathfrak{g}$-action on an additive category $\mathcal{C}$ is an action of the strict 2-category Rouquier denotes $\mathfrak{A}$; that is, it consists of

- a module category structure over the representations of the KLR algebra generated by functors $F_{i}$; that is, a functor $F \cong \oplus F_{i}$ such that $F^{k}$ carries an action of $R_{k}$. In particular, each of the functors $F_{i}$ carries a natural transformation $y$, usually denoted as a dot in literature such as [KL10, Weba, Webb], and

- right adjoints $E_{i}$ to these functors, such that

- the map Rouquier denotes $\rho_{s, \mu}$ in [Rou, $\left.\S 4.1 .3\right]$ is an isomorphism.

In particular, each pair of functors $E_{i}, F_{i}$ should be thought of a categorical $\mathfrak{s l}_{2}$ action in the sense of Chuang and Rouquier (in fact, they only consider categories which are abelian, artinian and noetherian, so small adjustments in the definition would be necessary). All 
other notions of categorification mentioned above are adding additional structure to this schema.

Since our main theorem will be a classification/uniqueness theorem, we need to have a notion of equivalence between categorical actions.

Definition 3.1. A strongly equivariant functor between two categories $\mathcal{C}_{1}, \mathcal{C}_{2}$ with categorical $\mathfrak{g}$-actions is

- a functor $\eta: \mathcal{C}_{1} \rightarrow \mathcal{C}_{2}$ together with

- isomorphisms of functors $F \eta \cong \eta F$ which commute with the $R_{k}$-actions on $F^{k} \eta \cong$ $\eta F^{k}$.

If we think of a categorical $\mathfrak{g}$-action as a representation of the 2-category $\mathfrak{A}$ in the strict 2 -category of $\mathbb{K}$-linear categories, this is the usual notion of natural transformation between representations of a 2-category.

We call such a functor a strongly equivariant equivalence if $\eta$ is an equivalence.

Our starting point is the categorification of $V$, an irreducible representation of $\mathfrak{g}$ with highest weight $\nu$. As mentioned in the introduction, there are several uniqueness theorems for such categorifications, based on work of Rouquier [Rou, §5.1]; since there are different contexts in which such representations appear, we record here the version that we require. We use $R^{\nu}$ to denote the cyclotomic KLR algebra for $\mathfrak{g}$ and the highest weight $\nu$ (for example, as discussed in Weba, $\S 1]$ ), and let $R_{k}^{\nu}$ to denote the finite dimensional subalgebra spanned by diagrams with $k$ strands, that is, the image of $R_{k}$.

Assume $\mathcal{C}$ is an artinian abelian $\mathbb{K}$-linear category.

Proposition 3.2. Assume $\mathcal{C}$ has a $\mathfrak{g}$-action by exact functors such that:

(1) the Grothendieck group $\mathbb{C} \otimes_{\mathbb{Z}} K^{0}(\mathrm{C})$ isomorphic to $V$;

(2) the subcategory $\mathcal{C}_{\nu}$ has a fixed equivalence to Vect $_{\mathbb{K}}$, sending $\mathbb{K}$ to an object $\mathbb{V}$;

(3) the transformation $y$ acts nilpotently on $F_{i}$.

Then $\mathcal{C}$ is strongly equivariantly equivalent to the category of finite-dimensional modules over $R^{\nu}$. The adjoint equivalences are given by

$$
\left(\bigoplus_{k} F^{k} \mathbb{V}\right) \otimes_{R^{\nu}} \bullet: R^{\nu}-\bmod \rightarrow \mathcal{C}, \quad \bigoplus_{k} \operatorname{Hom}\left(F^{k} \mathbb{V}, \bullet\right): \mathcal{C} \rightarrow R^{\nu}-\bmod
$$

Proof. The definition of a categorical action shows that the projective objects $F^{k} \mathbb{V}$ carry actions of the KLR algebra $R_{k}$. Note that we must have

$$
\operatorname{dim} \operatorname{Hom}\left(F_{i} \mathbb{V}, F_{i} \mathbb{V}\right)=\operatorname{dim} \operatorname{Hom}\left(\mathbb{V}, E_{i} F_{i} \mathbb{V}\right)=\alpha_{i}^{\vee}(\nu) .
$$

In fact, the categorification conditions imply that the monomials $1, y, \ldots, y^{\alpha_{i}^{\vee}\left(\nu_{j}\right)-1}$ span the algebra $\operatorname{Hom}\left(F_{i} \mathbb{V}, F_{i} \mathbb{V}\right)$; this is a special case of $\left.\mathbb{C L}, 3.12\right]$. Since $y$ is nilpotent, we must have

$$
\operatorname{Hom}\left(F_{i} \mathbb{V}, F_{i} \mathbb{V}\right) \cong \mathbb{K}[y] /\left(y^{\alpha_{i}^{\vee}(\nu)}\right) \cong R \alpha_{\alpha_{i}}^{\nu}
$$

This implies that the cyclotomic ideal for $\nu$ acts trivially on $F^{k} \mathbb{V}$.

Thus, if we consider the universal strongly equivariant functor from Rouquier's category $\mathcal{L}(\lambda)$ to $\mathcal{C}$, it factors through the category of projective $R^{\nu}$-modules, so the induced functor $F^{k} \mathbb{V} \otimes_{R_{k}^{\nu}} \bullet: R_{k}^{\nu}$-pmod $\rightarrow \mathcal{C}$ is strongly equivariant. Furthermore, since this functor is fully faithful on $\mathcal{C}_{\nu}$, this establishes that projective $R^{\nu}$-modules are the desired base change; in Rouquier's notation $R^{\nu}$-pmod $\cong \mathcal{L}(\lambda) \otimes_{\mathbb{K}} \operatorname{End}(\mathbb{V})$. Thus, this functor is fully faithful by [Rou, 5.4]. 
So, in order to show that we have an equivalence, we need only show that every indecomposable projective is a summand of $F^{k} \mathbb{V}$. Since summands of $F^{k} \mathbb{V}$ obtained using different monomials in the $F_{i}$ applied to $\mathbb{V}$ span the Grothendieck group $V$, there can be no others and we are done.

We'll note, this implies that in $\mathcal{C}$, every irreducible is absolutely irreducible, since this holds for the KLR algebra.

3.2. Definition. Let $V_{1}, \ldots, V_{n}$ be irreducible integrable $\mathfrak{g}$-modules with highest weights $\underline{\boldsymbol{\nu}}=\left(\nu_{1}, \ldots, \nu_{n}\right)$ and $\mathbb{K}$ be an infinite field; we let $\nu=\nu_{1}+\cdots+\nu_{n}$. We are going to define the notion of a categorification of the ordered tensor product $V_{1} \otimes V_{2} \otimes \ldots \otimes V_{n}$.

As before, let $\mathcal{C}$ be an abelian artinian $\mathbb{K}$-linear category, with each block equivalent to the representation category of a finite dimensional $\mathbb{K}$-algebra. The data of a tensor product categorification on $\mathcal{C}$ consists of two parts:

- a categorical $\mathfrak{g}$-action on $\mathcal{C}$ in the sense of Rouquier where the functors $E_{i}$ and $F_{i}$ are exact, and the natural transformation $y$ acts locally nilpotently on $F_{i}$ and,

- the structure of a standardly stratified category on $\mathcal{C}$ with poset $\Xi$.

These two pieces of data have to satisfy some compatibility conditions to be explained below.

(TPC1) The poset $\Xi$ is the set of $n$-tuples $\boldsymbol{\mu}=\left(\mu_{1}, \ldots, \mu_{n}\right)$, where $\mu_{i}$ is a weight of $V_{i}$. The poset structure is given by "inverse dominance order":

$$
\underline{\boldsymbol{\mu}}=\left(\mu_{1}, \ldots, \mu_{n}\right) \geqslant \underline{\boldsymbol{\mu}}^{\prime}=\left(\mu_{1}^{\prime}, \ldots, \mu_{n}^{\prime}\right) \text { if } \sum_{i=1}^{j} \mu_{i} \leqslant \sum_{i=1}^{j} \mu_{i}^{\prime}, \sum_{i=1}^{n} \mu_{i}=\sum_{i=1}^{n} \mu_{i} .
$$

As usual, we write $\beta_{1} \leqslant \beta_{2}$ if $\beta_{2}-\beta_{1}$ is a linear combination of positive roots with non-negative coefficients. We should note that the importance of order becomes immediately apparent in this definition.

(TPC2) The associated graded category gr $\mathcal{C}$ carries a categorical $\mathfrak{g}^{\oplus n}$-action with $K^{0}(\operatorname{gr} \mathcal{C}) \cong$ $V_{1} \otimes \cdots \otimes V_{n}$ as $\mathfrak{g}^{\oplus n}$-modules such that the weight $\boldsymbol{\mu}$ subcategory of gr $\mathcal{C}$ is precisely the subquotient $\mathcal{C}_{\leqslant \boldsymbol{\mu}} / \mathcal{C}_{\leq \boldsymbol{\mu}}$. We use ${ }_{j} E_{i}$ and ${ }_{j} F_{i}$ to denote categorification functors for the $j$ th copy of $\mathfrak{g}$. We assume that $\mathcal{C}_{\nu} \cong$ Vect $_{\mathbb{K}}$; we let $\mathbb{V}$ denote the unique indecomposable object in this subcategory. By Proposition 3.2, we thus have that $\operatorname{gr} \mathcal{C} \cong \mathcal{C}$, the representations of the cyclotomic KLR algebra of $\mathfrak{g}^{\oplus n}$ for the highest weight $\underline{\nu}$.

(TPC3) Finally, we must have a compatibility between the categorical $\mathfrak{g}$-action on $\mathcal{C}$ and the categorical $\mathfrak{g}^{\oplus n}$-action on gr $\mathcal{C}$ : for each $M \in \mathcal{C}_{\boldsymbol{\mu}}$, the object $E_{i} \Delta_{\mu}(M)$ admits a filtration with successive quotients being $\Delta\left({ }_{j} E_{i} M\right), \bar{j}=1, \ldots, n$. It is easy to see that such a filtration is determined uniquely, we call it a standard filtration on $E_{i} \Delta_{\boldsymbol{\mu}}(M)$.

Similarly, we require that $F_{i} \Delta(M)$ comes equipped with a filtration whose successive quotients are $\Delta\left({ }_{j} F_{i} M\right), j=1, \ldots, n$.

Since every irreducible is absolutely irreducible in $\operatorname{gr} \mathcal{C}$ as we noted above, this means that the same property will hold in any tensor product categorification.

Remark 3.3. Of course, we could try to make this definition more general by not requiring $y$ to be nilpotent; however, this would not really gain us any additional generality. If $y$ acted on the functor $F_{i}$ with more than one eigenvalue, it could then be split into generalized eigenspaces for $y$, and these functors would give a pair of categorical actions of $\mathfrak{g}$. Thus, 
we may as well assume that $y$ has only one eigenvalue $a$. Note that we can change this eigenvalue $a$ using the substitution $y \mapsto y-a$, at the cost of changing the relations of the KLR algebra. We must change the polynomials $Q_{i j}(u, v)$ by the same substitution.

Remark 3.4. Another point where the reader might wish to generalize this is to replace the condition that $\mathcal{C}_{\nu} \cong \operatorname{Vect}_{\mathbb{K}}$ with the condition that (say) $\mathcal{C}_{\nu}$ is the representation category of a local Artinian $\mathbb{K}$-algebra $A$. Our results should extend to this case, but at a considerable cost; in particular, the categorification obtained is no longer unique. Rather, the possible choices of $\operatorname{gr} \mathcal{C}$ will have moduli, given by considering the minimal polynomial of $y$ for its induced action on ${ }_{j} F_{i} \mathbb{V}$; the coefficients of this polynomial can be arbitrary elements of the radical of $A$, and one expects that there is a unique TPC with this choice of gr $\mathcal{C}$. Aside from the intrinsic nuisance of working relative to $A$, there are two relatively minor, but non-trivial, technical obstacles here:

- there are competing definitions of categorical $\mathfrak{g}$-action, and it's not clear that they give the same result. The classification mentioned above in terms of minimal polynomials is known for the Cautis-Lauda 2-category from [CL by [Weba, 1.12]; Rouquier has announced the same result for his 2-category, but the proof has yet to appear.

- it is not actually proven that a TPC will exist in this relative case since the corresponding algebras are not considered in Weba, though most results could be ported over by a careful use of Nakayama's Lemma.

As in any standardly stratified category, we have an isomorphism of Grothendieck groups $K^{0}(\operatorname{gr} \mathcal{C}) \cong K^{0}(\mathcal{C})$ via the map sending $[M] \mapsto[\Delta(M)]$. By assumption, we obtain an isomorphism $K^{0}(\mathcal{C}) \cong V_{1} \otimes \cdots \otimes V_{n}$. It follows immediately from (TPC3) that:

Proposition 3.5. For any tensor product categorification, this map is an isomorphism of $\mathfrak{g}$-modules.

We also note that any tensor product categorification is integrable, so by [Rou, 5.16], the functors $E_{i}$ and $F_{i}$ are necessarily biadjoint.

Remark 3.6. In fact, we could give an axiomatic description of a tensor product of arbitrary $\mathfrak{g}$-categorifications $\mathcal{C}^{1}, \ldots, \mathcal{C}^{n}$. Let us elaborate on this in the case when $n=2$. We say that a $\mathfrak{g}$-categorification $\mathcal{C}$ equipped with a standardly stratified structure is the tensor product $\mathcal{C}^{1} \otimes \mathcal{C}^{2}$ if

(TPC1') the poset $\Xi$ of $\mathcal{C}$ is the set of pairs $\left(\nu_{1}, \nu_{2}\right)$, where $\nu_{i}$ is a weight for $\mathcal{C}^{i}$, with the order given by $\left(\nu_{1}, \nu_{2}\right) \leqslant\left(\nu_{1}^{\prime}, \nu_{2}^{\prime}\right)$ if $\nu_{1} \geqslant \nu_{1}^{\prime}$ and $\nu_{1}+\nu_{2}=\nu_{1}^{\prime}+\nu_{2}^{\prime}$.

(TPC2') there is an identification of gr $\mathcal{C}$ with $\mathcal{C}^{1} \otimes \mathcal{C}^{2}$

(TPC3') for each $M \in \operatorname{gr} \mathcal{C}$ the object $E_{i} \Delta(M)$ admits a short exact sequence

$$
0 \longrightarrow \Delta\left(E_{i}^{1} M\right) \longrightarrow E_{i} \Delta(M) \longrightarrow \Delta\left(E_{i}^{2} M\right) \longrightarrow 0
$$

and similarly for the functors $F_{i}$.

Unfortunately, in general, we can prove neither existence nor uniqueness of such tensor products; we expect that it will arise from Rouquier's proposed internal tensor product.

3.3. Labeling. Before getting too deep into the structure of these categories, we should give a set of labels for simples (or indecomposable projectives) in $\mathcal{C}$.

As usual with a standardly stratified category, the simples (or indecomposable projectives) in $\mathcal{C}$ are in canonical bijection with the simples (or indecomposable projectives) in gr $\mathcal{C}$. Let $B\left(\nu_{j}\right)$ be the crystal of the irreducible representation $V_{j}$. By [LV11, §5.1], we have a canonical 
bijection between the product $B\left(\nu_{1}\right) \times \cdots \times B\left(\nu_{n}\right)$ and the set of simples (or indecomposable projectives) in gr $\mathcal{C}$. Recall that the set of simple objects in an arbitrary $\mathfrak{g}$-categorification has a $\mathfrak{g}$-crystal structure: if $L$ is a simple, then for $\tilde{e}_{i} L$ we take the head (equivalently, the socle) of the object $E_{i} L$ if the latter is nonzero and 0 else. The crystal operator $\tilde{f}_{i}$ is defined similarly using $F_{i}$; as usual, we use the notation ${ }_{j} \tilde{e}_{i},{ }_{j} \tilde{f}_{i}$ when considering these operators for $\mathfrak{g}^{\oplus n}$.

First consider the case of the categorification of a simple module with highest weight $\nu$, which, as before, we denote $\mathcal{C}$. One straightforward description of the projective for a crystal element uses the string parameterization of vertices of $B(\nu)$. Consider $\lambda \in B(\nu)$ for some $j=1, \ldots, n$, and let $P(\lambda)$ be the associated projective. Choose an infinite sequence $i_{1}, i_{2}, \ldots$ of nodes in the Dynkin diagram of $\mathfrak{g}$ containing each node infinitely many times. The string parameterization of $\lambda$ is the unique infinite sequence of integers $\left(a_{1}, a_{2}, \ldots\right)$ with almost all entries 0 such that

$$
\tilde{e}_{i_{j}}^{a_{j}} \cdots \tilde{e}_{1}^{a_{1}} \lambda \neq 0 \quad \tilde{e}_{i_{j}}^{a_{j}+1} \tilde{e}_{i_{j-1}}^{a_{j-1}} \cdots \tilde{e}_{1}^{a_{1}} \lambda=0 \quad \text { for all } j .
$$

We can order crystal elements by comparing string parametrizations lexicographically.

Proposition 3.7 (Khovanov-Lauda [KL09, 3.20]). The projective $P(\lambda)$ is the unique summand of $F_{i_{1}}^{a_{1}} F_{i_{2}}^{a_{2}} \cdots \mathbb{V}$ which doesn't appear in $F_{i_{1}}^{a_{1}^{\prime}} F_{i_{2}}^{a_{2}^{\prime}} \cdots \mathbb{V}$ for a word $\mathbf{a}^{\prime}$ larger than $\mathbf{a}$ in lexicographic order.

For $\mathfrak{g}^{\oplus n}$, we want to proceed a little differently; instead of applying this construction directly (which will work perfectly well), we compute the string parameterization of each component of $\underline{\boldsymbol{\lambda}}=\left(\lambda_{1}, \ldots, \lambda_{n}\right) \in B\left(\nu_{1}\right) \times \cdots \times B\left(\nu_{n}\right)$. Thus, we obtain $n$ different words $\mathbf{a}^{(1)}=\left(a_{1}^{(1)}, \ldots\right)$, etc. We can easily modify the proposition above to:

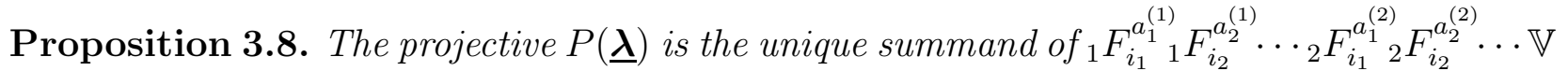
which doesn't appear in a corresponding monomial where any $\mathbf{a}^{(j)}$ is replaced by a larger word in lexicographic order.

3.4. Tensor product categorification on $\mathcal{C}^{o p p}$. Suppose that $\mathcal{C}$ is a tensor product categorification of $V_{1} \otimes \ldots \otimes V_{n}$ in the sense of the definition above. In this subsection we are going to prove that $\mathcal{C}^{o p p}$ is also a tensor product categorification of $V_{1} \otimes V_{2} \otimes \ldots \otimes V_{n}$.

Proposition 3.9. The category $\mathcal{C}^{o p p}$ is a tensor product categorification of $V_{1} \otimes \ldots \otimes V_{n}$

Proof. Conditions (TPC1) and (TPC2) are tautologically equivalent for $\mathcal{C}$ and $\mathcal{C}^{\text {opp }}$. Thus, we need only establish (TPC3).

We need to prove that $E_{i} \nabla_{\underline{\mu}}(M)$ has a filtration whose successive quotients are $\nabla_{\underline{\mu}+\alpha_{i}^{j}}\left({ }_{j} E_{i} M\right)$, and the analogous claim for $F_{i} \nabla_{\underline{\mu}}(M)$. Here $\alpha_{i}^{j}$ means the simple root $\alpha_{i}$ for the $j$ th copy of $\mathfrak{g}$.

First of all, $E_{i}, F_{i}$ preserve $\mathcal{C}^{\bar{\nabla}}, \mathcal{C}^{\nabla}$. This follows from the observation that $E_{i}, F_{i}$ preserve $\mathcal{C}^{\Delta}, \mathcal{C}^{\bar{\Delta}}$, combined with Lemma 2.4 and the biadjointness of $E_{i}$ and $F_{i}$.

Pick $N \in \mathcal{C}_{\underline{\boldsymbol{\mu}}^{\prime}}$, where $\underline{\boldsymbol{\mu}}^{\prime}-\underline{\boldsymbol{\mu}}=\alpha_{i}^{j}$. We see that

$$
\begin{aligned}
& \operatorname{Hom}_{\mathcal{C}}\left(\Delta_{\underline{\boldsymbol{\mu}}^{\prime}}(N), E_{i} \nabla_{\underline{\boldsymbol{\mu}}}(M)\right)=\operatorname{Hom}_{\mathcal{C}}\left(F_{i} \Delta_{\underline{\boldsymbol{\mu}}^{\prime}}(N), \nabla_{\underline{\boldsymbol{\mu}}}(M)\right)= \\
& =\operatorname{Hom}_{\mathcal{C}_{\underline{\boldsymbol{\mu}}}}\left({ }_{j} F_{i} N, M\right)=\bigoplus_{j=1}^{n} \operatorname{Hom}_{\mathcal{C}_{\underline{\mu}^{\prime}}}\left(N,{ }_{j} E_{i} M\right)= \\
& =\operatorname{Hom}_{\mathcal{C}}\left(\Delta_{\underline{\boldsymbol{\mu}}^{\prime}}(N), \nabla_{\underline{\boldsymbol{\mu}}^{\prime}}\left({ }_{j} E_{i} M\right)\right),
\end{aligned}
$$


where all equalities are natural isomorphisms of $\operatorname{End}_{\mathcal{C}_{\underline{\mu}^{\prime}}}(N)$-modules.

Now let us show that the claim in the beginning of the proof holds when $M$ is projective (=injective) in $\mathcal{C}_{\underline{\mu}}$. Recall that $E_{i} \nabla_{\underline{\mu}}(M) \in \mathcal{C}^{\nabla}$. In particular, if, in (3.1), for $N$ we take the simple in $\mathcal{C}_{\underline{\boldsymbol{\mu}}^{\prime}}$ labeled by $\lambda$, we see that the multiplicity of $\nabla(\lambda)$ in $E \nabla_{\underline{\mu}}(M)$ and $\bigoplus_{i=1}^{n} \nabla_{\underline{\boldsymbol{\mu}}+\alpha_{i}^{j}, \underline{\boldsymbol{\mu}}^{\prime}}\left({ }_{j} E_{i} \bar{M}\right)$ coincide. This implies the existence of a required filtration on $E_{i} \nabla_{\underline{\mu}}(M)$.

Proceed to the case of a general $M$. In this case, Lemma 2.4 just implies that $E_{j} \nabla(M) \in$ $\mathcal{C}^{\bar{\Delta}}$. So, by (2.1), the object $E_{j} \nabla(M)$ has a filtration with successive quotients $\nabla\left(M^{\prime}\right)$, $M^{\prime} \in \mathcal{C}_{\underline{\boldsymbol{\mu}}^{\prime}}$, for $\underline{\boldsymbol{\mu}}^{\prime}=\underline{\boldsymbol{\mu}}+\alpha_{i}^{j}, j=1, \ldots, n$. Recall that we write $\iota_{\underline{\boldsymbol{\mu}}^{\prime}}$ for the inclusion functor $\mathcal{C}_{\leqslant \underline{\boldsymbol{\mu}}^{\prime}} \hookrightarrow \mathcal{C}, \pi_{\underline{\mu}^{\prime}}$ for the projection functor $\mathcal{C}_{\leqslant \underline{\boldsymbol{\mu}^{\prime}}} \rightarrow \mathcal{C}_{\underline{\boldsymbol{\mu}}^{\prime}}$, and $\iota_{\underline{\boldsymbol{\mu}}^{\prime}}^{!}, \pi_{\underline{\boldsymbol{\mu}}^{\prime}}^{!}$for the left adjoint functors. Choose $j=1, \ldots, n$ and set $\underline{\boldsymbol{\mu}}^{\prime}=\underline{\boldsymbol{\mu}}+\alpha_{i}^{j}$. Consider the functor $\overline{\mathcal{F}}_{\underline{\boldsymbol{\mu}}^{\prime}}:=\pi_{\underline{\boldsymbol{\mu}}^{\prime}}^{!} \circ \pi_{\underline{\boldsymbol{\mu}}^{\prime}} \circ \iota_{\underline{\boldsymbol{\mu}}^{\prime}}^{!}: \mathcal{C} \rightarrow \mathcal{C}_{\leqslant} \underline{\boldsymbol{\mu}}^{\prime}$. It follows from Lemma 2.2 that the functor $\mathcal{F}_{\underline{\mu}^{\prime}}$ is exact on $\mathcal{C}^{\bar{\Delta}}$. It maps $M^{\prime} \in \mathcal{C}^{\bar{\Delta}}$ to the subquotient of the form $\Delta_{\underline{\mu}^{\prime}}\left(N^{\prime}\right)$, where $N^{\prime}=\bar{\pi}_{\underline{\mu}^{\prime}} \circ \iota_{\underline{\boldsymbol{\mu}}^{\prime}}^{!}\left(M^{\prime}\right)$. So we just need to prove that the functor $\pi_{\underline{\boldsymbol{\mu}}^{\prime}} \circ \iota_{\underline{\boldsymbol{\mu}}^{\prime}} \circ E_{i} \circ \Delta_{\underline{\mu}}: \mathcal{C}_{\underline{\boldsymbol{\mu}}} \rightarrow \mathcal{C}_{\underline{\boldsymbol{\mu}}^{\prime}}$ is isomorphic to ${ }_{j} E_{i}$ if $\underline{\boldsymbol{\mu}}^{\prime}=\underline{\boldsymbol{\mu}}+\alpha_{i}^{j}$ for some $j=1, \ldots, n$ and is zero else. The vanishing result follows from the form of $E_{i} \nabla_{\underline{\mu}}(M)$, for $M$ projective, obtained above. The isomorphism of functors follows from (3.1).

Remark 3.10. One can also equip the Ringel dual $\mathcal{C}^{\vee}$ with a categorical $\mathfrak{g}$-action turning $\mathcal{C}^{\vee}$ into a tensor product categorification of $V_{n} \otimes V_{n-1} \otimes \ldots \otimes V_{1}$, compare with [Losb, 7.1] and [Losc, 9.2]. Namely, using the identification $\left(\mathcal{C}^{\vee}\right)^{\bar{\Delta}} \cong \mathcal{C}^{\bar{\nabla}}$ one defines the categorification functors on $\left(\mathcal{C}^{\vee}\right)^{\bar{\Delta}}$ and then extends them to the whole category $\mathcal{C}^{\vee}$ obtaining a categorical $\mathfrak{g}$-action. Then it is not difficult to see that together with the standardly stratified structure on $\mathcal{C}^{\vee}$, this action becomes a tensor product categorification of $V_{n} \otimes V_{n-1} \otimes \ldots \otimes V_{1}$.

3.5. Relation with previous constructions. Concrete examples of categorical $\mathfrak{g}$-actions whose Grothendieck groups are tensor products have arisen in work in representation theory and topology.

Diagrammatic realizations. One obvious construction to compare the definition above with are the algebras $T \underline{\boldsymbol{\nu}}$ defined by the second author in [Weba, §2]. We refer the reader to that paper for the details of the definition. What is important for us is an inductive description of the representation categories of these algebras. Given the sequence of weights $\underline{\boldsymbol{\nu}}$, we define weights $\nu^{(k)}=\nu_{1}+\cdots+\nu_{k}$.

Attached to each $k$, we have an associated cyclotomic quotient of the KLR algebra

$$
R^{\nu^{(k)}}=R /\left\langle y_{1}^{\alpha_{i}^{\vee\left(\nu^{(k)}\right)}} e(\mathbf{i}) \mid \mathbf{i} \in I^{m}\right\rangle,
$$

equipped with projections $R^{\nu^{(k)}} \rightarrow R^{\nu^{(k-1)}}$, and induced inflation functors $\inf _{k}: R^{\nu^{(k-1)}}-\bmod \rightarrow$ $R^{\nu^{(k)}}-\bmod$.

Now, consider the category $\mathcal{C}(\underline{\boldsymbol{\nu}})$ defined as the category of representations of its category $\mathcal{C}(\underline{\boldsymbol{\nu}})$-proj of projectives (via the Yoneda embedding):

- we let $\mathcal{C}(\nu)$ just be the category of finite dimensional representations of $R^{\nu}$.

- The category of projectives $\mathcal{C}(\underline{\boldsymbol{\nu}})$-proj is the additive category of generated by sum-

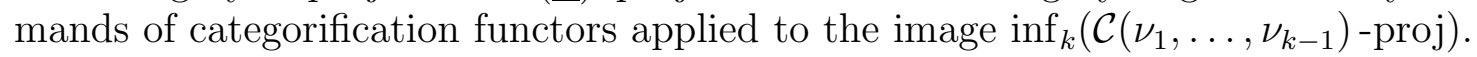

Thus, these are the minimal subcategories closed under the categorical $\mathfrak{g}$-action which contains the images of all inflation functors. 
Proposition 3.11. We have an equivalence $T^{\underline{\nu}}-\bmod \cong \mathcal{C}(\underline{\boldsymbol{\nu}})$.

Proof. This follows from [Weba, 3.24]. In that paper we define a fully faithful functor $T^{\boldsymbol{\nu}}$-pmod $\rightarrow R^{\nu}$-mod whose essential image is exactly the additive category generated by summands of $y_{\mathbf{i}, \nu} R^{\nu}$ for certain elements $y_{\mathbf{i}, \kappa}$ associated to sequences $\mathbf{i}=\left(i_{1}, \ldots, i_{m}\right)$ and functions $\kappa:[1, n] \rightarrow[0, m]$. If $\kappa(n) \neq m$, then $y_{\mathbf{i}, \kappa} T^{\nu}=F_{i_{m}}\left(y_{\mathbf{i}^{-}, \kappa} T^{\nu}\right)$ where $\mathbf{i}^{-}=\left(i_{1}, \ldots, i_{m-1}\right)$. Thus, the image of $T^{\underline{\nu}}$-mod is generated by categorification functors applied to the modules $y_{\mathbf{i}, \kappa} T^{\nu}$ with $\kappa(n)=m$. Since these modules are exactly obtained by applying the inflation functor inf ${ }_{n-1}$ to the images of $T^{\left(\nu_{1}, \ldots, \nu_{n-1}\right)}$-pmod, we are done.

The most important fact for us is that:

Theorem 3.12. The category $\mathcal{C}(\underline{\boldsymbol{\nu}})$ is a tensor product categorification for $V_{1} \otimes \cdots \otimes V_{n}$.

Proof. This category is a categorical $\mathfrak{g}$-module by [Weba, 2.11] and standardly stratified by Weba, 3.18]. (TPC1) is also part of the statement of [Weba, 3.18], (TPC2) follows from [Weba, 3.21] and (TPC3) follows from [Weba, 3.7-8].

Tensor product categorifications for $\mathfrak{s l}_{m}$ also arise in more classical representation theory. Here, we give two examples.

Category $\mathcal{O}$. Consider the Lie algebra $\mathfrak{g l}_{N}(\mathbb{C}$ ), its parabolic subalgebra with blocks (from top to bottom) of sizes $m_{1}, \ldots, m_{n}$ and also fix a positive integer $n$. Let $\mathcal{O}$ be the corresponding parabolic category $\mathcal{O}$. The integral blocks of this category form a highest weight category whose standard objects are parabolic Verma modules

$$
\Delta(\lambda) \text { with } \lambda=\left(\lambda_{1}, \cdots, \lambda_{N}\right) \text { for } \lambda_{1}>\lambda_{2}>\cdots>\lambda_{m_{1}}, \lambda_{m_{1}+1}>\cdots>\lambda_{m_{2}}, \cdots
$$

of highest weight $\rho+\sum \lambda_{i} \epsilon_{i}$.

Definition 3.13. Let $\mathcal{C}(\mathbf{m})$ be the the sum of blocks of $\mathcal{O}$ spanned by $\Delta(\lambda)$ with $\lambda_{i} \in$ $\{1, \ldots, m\}$.

This category is highest weight in the sense of [Losb] (more precisely, all categorical $\mathfrak{s l}_{2}$ actions corresponding to the simple roots are highest weight) and so is a tensor product categorification of $\bigwedge^{m_{1}} \mathbb{K}^{m} \otimes \bigwedge^{m_{2}} \mathbb{K}^{m} \otimes \cdots \otimes \bigwedge^{m_{n}} \mathbb{K}^{m}$. It was shown in [Weba, 4.2] that the category $\mathcal{C}$ is strongly equivariantly equivalent to $\mathcal{C}\left(\omega_{m_{1}}, \ldots, \omega_{m_{n}}\right)$, where $\omega_{i}$ is the $i$ th fundamental weight. The main theorem of this paper also provides a new proof of this equivalence.

Representations of $G L_{n}$. Often we can also realize tensor product categorifications as subquotients of interesting categories. Let us give an example when the field $\mathbb{K}$ has characteristic $p>0$ and the algebra $\mathfrak{g}$ acting is $\mathfrak{s l}_{p}$. Consider the category $\tilde{\mathcal{C}}=\bigoplus_{n=0}^{+\infty} \tilde{\mathcal{C}}_{n}$, where $\tilde{\mathcal{C}}_{n}$ is the category of polynomial representations of $\mathrm{GL}_{k}$ of degree $n \leqslant k$. This is a highest weight category, whose labeling poset is that of partitions (with respect to the $p$-dominance ordering).

A categorical $\hat{\mathfrak{s l}}_{p}$-action on this category was first introduced in [HY], and this action is highest weight in the sense of [Losb]. Fix a residue $r$ and consider the subalgebra $\mathfrak{s l}_{p} \subset \hat{\mathfrak{s l}}_{p}$ corresponding to the other $p-1$ residues.

We introduce an equivalence relation $\sim_{r}$ on the set of Young diagrams: $\lambda \sim_{r} \mu$ if the boxes in $\lambda$ and $\mu$ with residue $r$ are the same. Attached to each such equivalence class is a list of coordinates $\left(x_{0}, y_{0}\right),\left(x_{1}, y_{1}\right), \cdots,\left(x_{\ell}, x_{\ell}\right)$ given by the rightmost box in each diagonal of the partition diagram with content congruent to $r(\bmod p)$ listed left to right; we must 


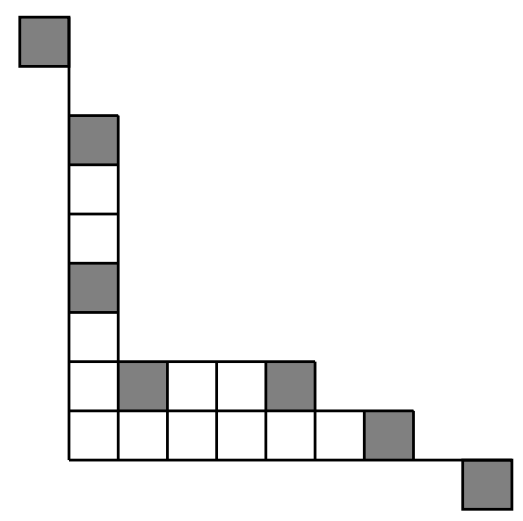

Figure 1 . The example with $p=3, r=0$ and $\lambda=\left(7,5,1^{5}\right)$. The boxes $\{(9,0),(7,1),(5,2),(2,2),(1,4),(1,7),(0,9)\}$ are marked.

also include the first empty diagonals encountered on the left and right, that is, we have $x_{0}=0$ and $y_{\ell}=0$. We let $m_{i}=y_{i}-y_{i+1}$, and note that $0 \leqslant m_{i} \leqslant p$.

Each equivalence class is an interval in the highest weight poset of $\tilde{\mathcal{C}}$, so for an equivalence class $e$, we can consider the subquotient category $\mathcal{C}_{e}$ corresponding to $e$. This is a highest weight category with a well-defined highest weight categorical action of $\mathfrak{s l}_{p}$ and a tensor product categorification of the product $\bigwedge^{m_{1}} \mathbb{K}^{p} \otimes \bigwedge^{m_{2}} \mathbb{K}^{p} \otimes \cdots \otimes \bigwedge^{m_{n}} \mathbb{K}^{p}$.

For example, if $p=3, r=0$ and $\lambda=\left(7,5,1^{5}\right)$, we have the sequence of boxes

$$
\{(9,0),(7,1),(5,2),(2,2),(1,4),(1,7),(0,9)\}
$$

and the tensor product categorified is $\bigwedge^{2} \mathbb{K}^{3} \otimes \bigwedge^{2} \mathbb{K}^{3} \otimes \mathbb{K}^{3} \otimes \mathbb{K}^{3}$.

Quotient categories. When $\mathfrak{g}$ is $\widehat{\mathfrak{s l}}_{m}$, then by Weba, 4.14] the category $\mathcal{C}\left(\omega_{m_{1}}, \ldots, \omega_{m_{n}}\right)$ is equivalent to an appropriate quotient of the category of representations of cyclotomic $q$-Schur algebras, where $q$ is a primitive $m$ th root of 1 , with parameters corresponding to the residues $m_{1}, \ldots, m_{n}$. However, it is not so easy to see the structure of a tensor product categorification on the latter from the beginning, this should require an appropriate modification of the splitting procedure explained below.

Also we would like to point out that tensor product categorifications for non-fundamental weights can be realized as quotients of parabolic categories $\mathcal{O}$ (for finite type $A$ ) or of the representation categories of cyclotomic Schur algebras. Again, it is easier to see this if one works with categories $\mathcal{C}\left(\nu_{1}, \ldots, \nu_{n}\right)$.

Proposition 3.14. For any tensor product categorification $\mathcal{C}\left(\nu_{1}, \ldots, \nu_{n}\right)$, and list of indices $1 \leqslant j_{1}<\cdots<j_{m}<n$, we have an exact quotient functor

$$
p_{\mathbf{j}}: \mathcal{C}\left(\nu_{1}, \ldots, \nu_{n}\right) \rightarrow \mathcal{C}\left(\nu_{1}+\cdots+\nu_{j_{1}}, \nu_{j_{1}+1}+\cdots+\nu_{j_{2}}, \ldots, \nu_{j_{m}+1}+\cdots+\nu_{n}\right)
$$

categorifying the natural projection of tensor product representations.

Proof. By [Weba, 2.7], there's an idempotent $e_{\mathbf{j}}$ in $T^{\underline{\nu}}$ such that $e_{\mathbf{j}} T^{\underline{\nu}} e_{\mathbf{j}} \cong T^{\underline{\mathbf{j}}} \mathbf{j}$ where $\underline{\boldsymbol{\nu}}_{\mathbf{j}}=$ $\left(\nu_{1}+\cdots+\nu_{j_{1}}, \ldots, \nu_{j_{m}+1}+\cdots+\nu_{n}\right)$. This is the sum of the diagrams with no crossings, no dots, and no black strands between the red strands corresponding to weights we have condensed.

Thus, the desired exact functor $T^{\underline{\nu}}-\bmod \rightarrow T^{\underline{\underline{\nu}}}$ - $\bmod$ is just multiplication by $e_{\mathbf{j}}$. 


\section{Categorical splitting}

4.1. Setting. As before, fix simple $\mathfrak{g}$-modules $V_{i}$ with highest weight $\nu_{i}$. Consider a tensor product categorification $\mathcal{C}$ of $V_{1} \otimes V_{2} \otimes \cdots \otimes V_{n}$ with poset $\Xi=\left\{\underline{\boldsymbol{\mu}}=\left(\mu_{1}, \ldots, \mu_{n}\right)\right\}$. Set $\Xi_{0}:=\left\{\mu \in \Xi \mid \mu_{n}<\nu_{n}\right\}$. We intend to study the subcategory $\mathcal{C}_{\Xi_{0}}$ and the corresponding quotient $\underline{\mathcal{C}}^{+}:=\mathcal{C} / \mathcal{C}_{\Xi_{0}}$. We remark that, under our identification $[\mathcal{C}] \cong V_{1} \otimes V_{2} \otimes \cdots \otimes V_{n}$, the space $\left[\underline{\mathcal{C}}^{+}\right]$is naturally identified with $V_{1} \otimes V_{2} \otimes \cdots \otimes V_{n-1} \otimes v_{\nu_{n}}$, where $v_{\nu_{n}}$ is the singular vector in $V_{n}$. We write $\mathcal{C}^{i}$ for the categorification of the simple $V_{i}$. Further, for $\lambda_{i} \in B\left(\nu_{i}\right)$, by $v_{\lambda_{i}}$ we denote the class of the corresponding simple object in $\left[\mathcal{C}^{i}\right]=V_{i}$.

Our goal in this section is to produce a categorical $\mathfrak{g}$-action on the quotient $\underline{\mathcal{C}}^{+}:=\mathcal{C} / \mathcal{C}_{\Xi_{0}}$ making it into a tensor product categorification of $V_{1} \otimes V_{2} \otimes \cdots \otimes V_{n-1}$. Since $\Xi_{0}$ is a poset ideal, the quotient category has a natural standardly stratified structure that we are going to use. The corresponding poset will be denoted by $\Xi^{+}$, the poset associated to $V_{1} \otimes V_{2} \otimes \cdots \otimes V_{n-1}$ in the same way as $\Xi$ is associated to $V_{1} \otimes V_{2} \otimes \cdots \otimes V_{n}$.

The following lemma shows that half of categorification functors act in a straight-forward way on $\underline{\mathcal{C}}^{+}$.

Lemma 4.1. The subcategory $\mathcal{C}_{\Xi_{0}}$ is stable with respect to the functors $F_{i}$.

Proof. Any simple in $\mathcal{C}_{\Xi_{0}}$ is a composition factor of $\Delta(\lambda)$ with $\varrho(\lambda) \in \Xi_{0}$. So it is enough to show that $F_{i} \Delta(\lambda) \in \mathcal{C}_{\Xi_{0}}$. This is an easy corollary of (TPC3).

So we have the induced functor $\underline{F}_{i}^{+}$on $\underline{\mathcal{C}}^{+}$. The same condition (TPC3) shows that, in general, $\mathcal{C}_{\Xi_{0}}$ is not closed with respect to $E_{i}$. In order to get the functor $\underline{E}_{i}^{+}$on $\underline{\mathcal{C}}^{+}$we will need to truncate the functor $E_{i}$. Our construction will generalizes [Losb, Section 5], where the first author studied essentially the case of the tensor products of the tautological $\mathfrak{s l}_{2}$-modules.

4.2. An equivalence. We will construct the functors $\underline{E}_{i}^{+}$one simple root at a time. Thus, throughout the remainder of this section, we fix an index $i \in I$.

First, we are going to take a minimal, in a way, standardly stratified quotient of $\mathcal{C}$, where $E_{i}$ is well-defined. Namely, consider $\Xi_{1}=\left\{\underline{\boldsymbol{\mu}} \in \Xi \mid \nu_{n}-\mu_{n} \notin \mathbb{Z} \alpha_{i}\right\}$, clearly, $\Xi_{1}$ is a poset ideal contained in $\Xi_{0}$. An analogous argument to Lemma 4.1 shows that:

Lemma 4.2. The subcategory $\mathcal{C}_{\Xi_{1}}$ is stable with respect to both $E_{i}, F_{i}$ and so we have welldefined functors on $\underline{\mathcal{C}}\left(=\underline{\mathcal{C}}_{i}\right):=\mathcal{C} / \mathcal{C}_{\Xi_{1}}$ again denoted by $E_{i}, F_{i}$.

Set $\Xi=\Xi / \Xi_{1}$, this is the poset of the standardly stratified category $\underline{\mathcal{C}}$. We will need a standardly stratified subcategory $\underline{\mathcal{C}}^{-}\left(=\underline{\mathcal{C}}_{i}^{-}\right)$inside $\underline{\mathcal{C}}$. Namely, consider the poset ideal $\Xi^{-} \subset$ $\Xi$ consisting of all $\underline{\boldsymbol{\mu}}$ with $\mu_{n}=s_{i} \nu_{n}$, where $s_{i}$ is the simple reflection corresponding to $i$. Let $\underline{\mathcal{C}}^{-} \subset \underline{\mathcal{C}}$ be the subcategory corresponding to $\underline{\Xi}^{-}$. Under our identification $[\mathcal{C}] \cong V_{1} \otimes \ldots \otimes V_{n}$, the complexified Grothendieck group $\left[\underline{\mathcal{C}}^{-}\right]$is identified with $V_{1} \otimes V_{2} \otimes \ldots \otimes V_{n-1} \otimes v_{s_{n} \mu_{n}}$. Let $\iota$ be the inclusion $\underline{\mathcal{C}}^{-} \hookrightarrow \underline{\mathcal{C}}$, and $\pi: \underline{\mathcal{C}} \rightarrow \underline{\mathcal{C}}^{+}$be the projection.

Proposition 4.3. The functor $\mathcal{E}=\pi \circ E_{i}^{(r)} \circ \iota: \underline{\mathcal{C}}^{-} \rightarrow \underline{\mathcal{C}}^{+}$is an equivalence of standardly stratified categories with quasi-inverse given by $\mathcal{F}:=\iota^{!} \circ F_{i}^{(r)} \circ \pi^{!}$.

The proof closely follows that in [Losb, Section 5.2] but we are going to provide it for readers convenience. So far, we notice that, by the construction, $\mathcal{E}$ is exact and $\mathcal{F}$ is left adjoint to $\mathcal{E}$.

We start by establishing some basic properties of $\mathcal{E}$, compare with [Losb, Lemma 5.1]. Thanks to results of Chuang and Rouquier, [CR08], for $\underline{\boldsymbol{\mu}} \in \Xi^{-}$the functors ${ }_{n} E_{i}^{(r)}{ }_{n} F_{i}^{(r)}$ 
restrict to quasi-inverse equivalences between $\mathcal{C}_{s_{i} \nu_{n}}^{n}, \mathcal{C}_{\nu_{n}}^{n}$. We will identify these categories using the functors. Also we identify the posets $\Xi^{ \pm}$with the poset associated to $V_{1} \otimes \ldots \otimes V_{n-1}$. Finally, we identify $\left[\underline{\mathcal{C}}^{ \pm}\right]$with $V_{1} \otimes V_{2} \otimes \ldots \otimes V_{n-1}$ by sending $\bar{\Delta}\left(\lambda_{1}, \ldots, \lambda_{n-1}, \lambda_{n}\right)$ (where, recall, $\lambda_{n}$ is the label of the only simple object in $\mathcal{C}_{\nu_{n}}^{n}$ or $\mathcal{C}_{s_{i} \nu_{n}}^{n}$ ) to $v_{\lambda_{1}} \otimes \ldots \otimes v_{\lambda_{n-1}}$. Below for $\underline{\boldsymbol{\lambda}}=\left(\lambda_{1}, \ldots, \lambda_{n-1}\right)$ we write $L_{ \pm}(\underline{\boldsymbol{\lambda}}), \Delta_{ \pm}(\underline{\boldsymbol{\lambda}})$ etc. for the corresponding objects in $\underline{\mathcal{C}}^{ \pm}$.

\section{Lemma 4.4.}

(1) The functor $\mathcal{E}$ intertwines the standardization functors $\Delta_{\underline{\mu}}$, where $\underline{\boldsymbol{\mu}}=\left(\mu_{1}, \ldots, \mu_{n-1}\right)$.

(2) The functor $\mathcal{E}$ intertwines the costandardization functors $\nabla_{\underline{\mu}}$.

(3) The induced map $[\mathcal{E}]:\left[\underline{\mathcal{C}}^{-}\right] \rightarrow\left[\underline{\mathcal{C}}^{+}\right]$is the identity.

(4) $\mathcal{E}\left(L_{-}(\lambda)\right)=L_{+}(\lambda)$.

Proof. Let us prove (1). Set $\underline{\boldsymbol{\mu}}_{+}:=\left(\mu_{1}, \ldots, \mu_{n-1}, s_{i} \nu_{n}\right), \underline{\boldsymbol{\mu}}_{-}:=\left(\mu_{1}, \ldots, \mu_{n-1}, \nu_{n}\right)$. The object $E_{i}^{r} \Delta_{\underline{\mu}_{-}}(N)$ is $\bar{\Delta}$-filtered. Moreover, applying condition (TPC3) in the definition of a tensor product categorification $r$ times we get a filtration on $E_{i}^{r} \Delta_{\underline{\mu}_{-}}(N)$ whose successive quotients looks as follows: $\Delta_{\underline{\mu}_{-}+\alpha}\left(E_{i}^{\alpha} N\right)$, where $\alpha=\sum_{j=1}^{n} m_{j} \alpha_{i}^{j}$ with $\sum m_{j}=r$, and $E_{i}^{\alpha}:={ }_{1} E_{i}^{m_{1}} \otimes$ ${ }_{2} E_{i}^{m_{2}} \otimes \ldots{ }_{n} E_{i}^{m_{n}}$, and that quotient appears $r !\left(\begin{array}{c}r \\ m_{1}, \ldots, m_{n}\end{array}\right)$ times. The only quotient that survives under $\pi$ is the subobject $\Delta_{\underline{\mu}_{+}}\left({ }_{n} E_{i}^{r} N\right)$. The functor $\pi \circ E_{i}^{r} \circ \iota$ is isomorphic to $\mathcal{E}^{\oplus r !}$, and ${ }_{n} E_{i}^{r}$ is isomorphic to the sum of $r$ ! copies of our identification $\mathcal{C}_{\underline{\mu}_{-}} \hookrightarrow \mathcal{C}_{\underline{\mu}_{+}}$. We deduce that the functors $\mathcal{E} \circ \Delta_{\underline{\mu},-}^{\oplus r !}$ and $\Delta_{\underline{\mu},+}^{\oplus r !}$ are isomorphic. We claim that this implies that $\mathcal{E} \circ \Delta_{\underline{\mu},-} \cong \Delta_{\underline{\mu},+}$. It is enough to prove an isomorphism on each block separately. Since all weight spaces in $V_{1} \otimes \ldots \otimes V_{n-1}$ are finite dimensional, the blocks of $\underline{\mathcal{C}}^{ \pm}$are isomorphic to categories of finite dimensional modules of finite dimensional algebras. The functors $\mathcal{E} \circ \Delta_{\underline{\mu},-}, \Delta_{\boldsymbol{\mu},+}$ are right exact and so are given by tensor products with bimodules, say $B_{1}, B_{2}$. We know that $B_{1}^{\oplus r !} \cong B_{2}^{\oplus r !}$ and hence, by the Krull-Schmidt theorem, $B_{1} \cong B_{2}$.

(2) follows from (1) applied to $\mathcal{C}^{o p p}$ or can be proved completely analogously to (1). (3) is a direct corollary of $(1)$ and the particular form of the identification $\left[\underline{\mathcal{C}}^{+}\right] \cong\left[\underline{\mathcal{C}}^{-}\right]$. To prove (4) we notice that $L_{-}(\lambda)$ is the image of any nonzero morphism $\varphi: \bar{\Delta}_{-}(\lambda) \rightarrow \bar{\nabla}_{-}(\lambda)$. Since $\mathcal{E}$ is exact, we see that $\mathcal{E}\left(L_{-}(\lambda)\right)$ is the image of $\mathcal{E}(\varphi): \mathcal{E}\left(\bar{\Delta}_{-}(\lambda)\right) \rightarrow \mathcal{E}\left(\bar{\nabla}_{-}(\lambda)\right)$. Thanks to (1) and $(2), \mathcal{E}\left(\bar{\Delta}_{-}(\lambda)\right)=\bar{\Delta}_{+}(\lambda), \mathcal{E}\left(\bar{\nabla}_{-}(\lambda)\right)=\bar{\nabla}_{+}(\lambda)$. So $\mathcal{E}\left(L_{-}(\lambda)\right)$ is either $L_{+}(\lambda)$ or 0 . The latter is impossible because of $(3)$.

Now let us list some basic properties of $\mathcal{F}$, compare with [Losb, Lemmas 5.2,5.3].

\section{Lemma 4.5.}

(1) We have an isomorphism $\mathcal{F}\left(P_{+}(\underline{\boldsymbol{\lambda}})\right) \cong P_{-}(\underline{\boldsymbol{\lambda}})$ for any $\underline{\boldsymbol{\lambda}}=\left(\lambda_{1}, \ldots, \lambda_{n-1}\right)$.

(2) The natural morphism $\mathcal{F} \circ \mathcal{E}(M) \rightarrow M$ is surjective for any $M \in \underline{\mathcal{C}}^{-}$.

(3) The functors $\mathcal{F}\left(\Delta_{\underline{\mu},+}(\bullet)\right)$ and $\Delta_{\underline{\mu},-}(\bullet)$ are isomorphic.

(4) The natural morphism $\mathcal{F} \circ \mathcal{E}(M) \rightarrow M$ is an isomorphism for $M \in\left(\underline{\mathcal{C}}^{-}\right)^{\bar{\Delta}}$.

Proof. Being a left adjoint of the exact functor $\mathcal{E}$, the functor $\mathcal{F}$ maps projectives to projectives. Since, thanks to Lemma 4.4, $\mathcal{E}\left(L_{-}(\lambda)\right)=L_{+}(\lambda),(1)$ follows.

To prove $(2)$ we notice that the cokernel of $\mathcal{F} \circ \mathcal{E}(M) \rightarrow M$ vanishes under $\mathcal{E}$. Thanks to (3) of Lemma 4.4, this implies that the cokernel is 0.

Let us prove (3). Similarly to the proof of (1) in Lemma 4.4, it is enough to show that the functors $\mathcal{F} \circ \Delta_{\underline{\mu},+}(\bullet)^{\oplus r !}=\iota^{!} \circ F_{i}^{r} \circ \pi^{!} \circ \Delta_{\underline{\mu}_{+}}(\bullet)$ and $\Delta_{\underline{\mu},-}(\bullet)^{\oplus r !}=\Delta_{\underline{\mu}_{-}}\left(\otimes_{i=1}^{n-1} 1_{\mathcal{C}_{\mu_{i}}^{i}} \otimes{ }_{n} F_{i}^{r}(\bullet)\right)$ are isomorphic. We have $\pi^{!} \circ \Delta_{\underline{\mu},+}=\Delta_{\underline{\mu}}$. Then thanks to condition (TPC3) for $F_{i}$ applied 
$r$ times, we see that $F_{i}^{r} \circ \Delta_{\underline{\mu}}(M)$ has a filtration with successive quotients of the form $\Delta_{\underline{\mu}-\alpha}\left(F_{i}^{\alpha} M\right)$, each occurring with multiplicity $r !\left(\begin{array}{c}r \\ m_{1}, \ldots, m_{n}\end{array}\right)$ (our notation is the same as in the proof of Lemma 4.4). The only quotient lying in $\underline{\mathcal{C}}_{-}$is the top quotient, it is naturally identified with $\Delta_{\underline{\mu},-}(\bullet)^{\oplus r !}$. The image of $\Delta_{\underline{\mu}-\alpha}\left(F_{i}^{\alpha} M\right)$ under $\iota^{!}$coincides with the top quotient and so (3) is proved.

Let us prove (4). Thanks to (3) and (1) of Lemma 4.4, the morphism $\mathcal{F} \circ \mathcal{E}(\bar{\Delta}(\lambda)) \rightarrow \bar{\Delta}(\lambda)$ is an isomorphism. Now the proof repeats that of [Losb, Lemma 5.3(2)] (we remark that there the notation is different: $\mathcal{F}$ denotes an exact functor, while $\mathcal{E}$ is its left adjoint).

As in [Losb], to show that $\mathcal{E}$ is an equivalence, it remains to prove the following result.

Lemma 4.6. $\mathcal{E} P_{-}(\lambda)=P_{+}(\lambda)$ for all $\lambda$, and $\mathcal{E}$ is fully faithful on $\underline{\mathcal{C}}^{-}-$proj.

Proof. We have an identification

$$
\sigma: \operatorname{Hom}_{\underline{\mathcal{C}}^{+}}\left(P_{+}(\lambda), \mathcal{E} P_{-}(\lambda)\right)=\operatorname{Hom}_{\underline{\mathcal{C}}^{-}}\left(\mathcal{F} P_{+}(\lambda), P_{-}(\lambda)\right)=\operatorname{End}_{\underline{\mathcal{C}}^{-}}\left(P_{-}(\lambda), P_{-}(\lambda)\right) .
$$

We want to prove that $\varphi:=\sigma^{-1}(\mathrm{id})$ is an isomorphism. We have $\sigma(\varphi)=\eta \circ \mathcal{F} \varphi \circ \theta$, where $\eta$ is a natural morphism $\mathcal{F E} P_{-}(\lambda) \rightarrow P_{-}(\lambda)$ that is an isomorphism by (4) of Lemma 4.5, and $\theta$ is an isomorphism $\mathcal{F} P_{+}(\lambda) \stackrel{\sim}{\rightarrow} P_{-}(\lambda)$ from $(1)$ of Lemma 4.5 .

We claim that $P_{+}(\lambda)$ and $P_{-}(\lambda)$ have the same classes in $\left[\underline{\mathcal{C}}^{+}\right]=\left[\underline{\mathcal{C}}^{-}\right]$. By Lemma 2.5 to check this it suffices to show that $\left[\bar{\nabla}_{+}\left(\lambda^{\prime}\right): L_{+}(\lambda)\right]=\left[\bar{\nabla}_{-}\left(\lambda^{\prime}\right): L_{-}(\lambda)\right]$. The latter follows from (2)-(4) of Lemma 4.6. The same lemma now implies that the classes of $P_{+}(\lambda), \mathcal{E} P_{-}(\lambda)$ in $\underline{\mathcal{C}}^{+}$coincide. So, as in [Losb, Lemma 5.4], it is enough to prove that $\varphi$ is surjective.

Assume the converse, let $P_{+}(\lambda) \stackrel{\varphi}{\rightarrow} \mathcal{E} P_{-}(\lambda)$ have a nontrivial cokernel, say $K$. Applying $\mathcal{F}$ to the exact sequence

$$
P_{+}(\lambda) \stackrel{\varphi}{\rightarrow} \mathcal{E} P_{-}(\lambda) \rightarrow K \rightarrow 0
$$

we get an exact sequence

$$
\mathcal{F} P_{+}(\lambda) \stackrel{\mathcal{F} \varphi}{\longrightarrow} \mathcal{F E} P_{-}(\lambda) \rightarrow \mathcal{F} K \rightarrow 0
$$

But, being a composition of isomorphisms, $\mathcal{F} \varphi$ is an isomorphism itself. So $\mathcal{F} K=0$. It follows that $\operatorname{Hom}_{\mathcal{C}^{-}}\left(\mathcal{F} K, L_{-}\left(\lambda^{\prime}\right)\right)=0$ for any $\lambda^{\prime}$. But the last $\operatorname{Hom}$ is $\operatorname{Hom}_{\mathcal{C}^{+}}\left(K, \mathcal{E} L_{-}\left(\lambda^{\prime}\right)\right)=$ $\operatorname{Hom}_{\underline{\mathcal{C}}^{+}}\left(K, L_{+}\left(\lambda^{\prime}\right) \overline{)}\right.$. Since the latter is 0 for all $\lambda^{\prime}$ we deduce that $K$ is zero. This completes the proof of $\mathcal{E} P_{-}(\lambda)=P_{+}(\lambda)$.

The full faithfulness follows from

$$
\begin{aligned}
& \operatorname{Hom}_{\underline{\mathcal{C}}^{-}}\left(P_{-}(\lambda), P_{-}\left(\lambda^{\prime}\right)\right)=\operatorname{Hom}_{\underline{\mathcal{C}}^{-}}\left(\mathcal{F} P_{+}(\lambda), P_{-}\left(\lambda^{\prime}\right)\right)= \\
& \operatorname{Hom}_{\underline{\mathcal{C}}^{+}}\left(P_{+}(\lambda), \mathcal{E} P_{-}\left(\lambda^{\prime}\right)\right)=\operatorname{Hom}_{\underline{\mathcal{C}}^{+}}\left(P_{+}(\lambda), P_{+}(\lambda)\right) .
\end{aligned}
$$

Lemma 4.7. We have $\mathcal{F} \circ F_{i} \cong F_{i} \circ \mathcal{F}$ and $\mathcal{E} \circ F_{i} \cong F_{i} \circ \mathcal{E}$.

Proof. Since we have already checked that $\mathcal{F}, \mathcal{E}$ are mutually quasi-inverse equivalences, it is enough to show that $\mathcal{E} \circ F_{i} \cong F_{i} \circ \mathcal{E}$. We have $\mathcal{E} \circ F_{i}=\pi \circ E_{i}^{(r)} \circ \iota \circ F_{i} \cong \pi \circ E_{i}^{(r)} F_{i} \circ \iota$ (because $F_{i} \circ \iota \cong \iota \circ F_{i}$ ) and $F_{i} \circ \mathcal{E} \cong \pi \circ F_{i} E_{i}^{(r)} \circ \iota$. As above, it is enough to check that $\pi \circ E_{i}^{r} F_{i} \circ \iota \cong \pi \circ F_{i} E_{i}^{r} \circ \iota$. Also it is enough to do this blockwise. But every block lies in a weight subcategory of $\mathcal{C}$. By [CR08, on a weight subcategory, we have an isomorphism $F_{i} E_{i}^{r} \oplus\left(E_{i}^{r-1}\right)^{\oplus d_{1}} \cong E_{i}^{r} F_{i} \oplus\left(E_{i}^{r-1}\right)^{\oplus d_{2}}$, where $d_{1}, d_{2}$ are non-negative integers. Repeating the argument of the proof of (1) in Lemma 4.4, we see that $\pi \circ E_{i}^{r-1} \circ \iota=0$. 
4.3. Functor $\underline{E}_{i}$. Recall that the subcategory $\underline{\mathcal{C}}^{-} \subset \underline{\mathcal{C}}$ is closed under $F_{i}$, let $\underline{F}_{i}$ denote the restriction of $F_{i}$ to $\underline{\mathcal{C}}^{-}$. The functor $\underline{F}_{i}$ has both a left adjoint $F_{i}^{!}$, and a right adjoint $F_{i}^{*}$. They are obtained as

$$
F_{i}^{!}=\iota^{!} \circ E_{i} \circ \iota \quad F_{i}^{*}=\iota^{*} \circ E_{i} \circ \iota,
$$

where $\iota$ is the inclusion functor $\underline{\mathcal{C}}^{-} \hookrightarrow \underline{\mathcal{C}}$, and $\iota^{!}, \iota^{*}$ are its left and right adjoints. To produce a functor $\underline{E}_{i}$ on $\underline{\mathcal{C}}^{-}$that, together with $\underline{F}_{i}$, will equip $\underline{\mathcal{C}}^{-}$with a categorical $\mathfrak{s l}_{2}$-action, it is enough to show that $F_{i}^{!} \cong F_{i}^{*}$.

We will approach this problem in a way analogous to [Losb]: we will show that both $F_{i}^{!}$ and $F_{i}^{*}$ will be isomorphic to the third functor, $\underline{E}_{i}:=\mathcal{F} \circ \pi \circ E_{i}^{(r+1)} \circ \iota$.

Lemma 4.8. We have isomorphisms of functors $F_{i}^{!} \cong \underline{E}_{i} \cong F_{i}^{*}$.

We note that this proof closely follows [Losb, 5.3].

Proof. If we prove the first equality, the second will follow by symmetry, applying the theorem to $\mathcal{C}^{\text {opp }}$.

It is sufficient to prove that $\mathcal{E} \circ \underline{E}_{i}=\pi \circ E_{i}^{(r+1)} \circ \iota: \underline{\mathcal{C}}^{-} \rightarrow \underline{\mathcal{C}}^{+}$is isomorphic to $\mathcal{E} \circ \underline{F}_{i}^{!}=$ $\pi \circ E_{i}^{(r)} \circ \iota \iota^{!} \circ E_{i} \circ \iota$. Consider the adjunction epimorphism $\operatorname{id}_{\underline{\mathcal{C}}} \rightarrow \iota \iota^{!}$. Composing it with $\pi \circ E_{i}^{(r)}$ on the left and $E_{i} \circ \pi^{!}$on the right, we get an epimorphism

$$
\pi \circ E_{i}^{(r)} E_{i} \circ \iota \rightarrow \mathcal{E} \circ \underline{F}_{i}^{!}
$$

Recall that we have a decomposition $E_{i}^{(r)} E_{i} \cong \mathbb{K}^{r+1} \otimes E_{i}^{(r+1)}$. Picking a vector space embed$\operatorname{ding} \mathbb{K} \hookrightarrow \mathbb{K}^{r+1}$, we get a functor morphism

$$
\pi \circ E_{i}^{(r+1)} \circ \iota \rightarrow \mathcal{E} \circ \underline{F}_{i}^{!}
$$

We need to check that, for each given weight subcategory, there is an embedding $\mathbb{K} \hookrightarrow \mathbb{K}^{r+1}$ that makes the corresponding morphism (4.2) an isomorphism.

It is enough to check that there is an embedding $\mathbb{K} \hookrightarrow \mathbb{K}^{r+1}$ such that (4.2) is an isomorphism on all standardly filtered objects (because all functors under consideration are right exact). This reduces to checking that (4.2) is an isomorphism on all objects $\Delta_{\underline{\mu}-}(N)$ in a given weight subcategory as all functors under consideration are exact on standardly filtered objects (the embedding $\mathbb{K} \hookrightarrow \mathbb{K}^{r+1}$ may depend on $\sum_{i=1}^{n-1} \mu_{i}$ ). This, in its turn, boils down to checking that there is an embedding $\mathbb{K} \hookrightarrow \mathbb{K}^{r+1}$ such that the composed morphism

$$
\rho_{\underline{\boldsymbol{\mu}}^{\prime},+} \circ E_{i}^{(r+1)} \Delta_{\underline{\boldsymbol{\mu}},-} \hookrightarrow \rho_{\underline{\boldsymbol{\mu}}^{\prime},+} E_{i}^{(r)} E_{i} \Delta_{\underline{\boldsymbol{\mu}},-} \rightarrow \rho_{\underline{\boldsymbol{\mu}}^{\prime},+} E_{i}^{(r)} \circ F_{i}^{!} \Delta_{\underline{\boldsymbol{\mu}},-}
$$

is an isomorphism of functors $\underline{\mathcal{C}}_{\boldsymbol{\mu}}^{-} \rightarrow \underline{\mathcal{C}}_{\boldsymbol{\mu}^{\prime}}^{+}$. Here $\rho_{\underline{\boldsymbol{\mu}}^{\prime},+}$ is the functor $\underline{\mathcal{C}}^{\bar{\Delta}} \rightarrow \mathcal{C}_{\left(\underline{\boldsymbol{\mu}}^{\prime}, \nu_{n}\right)}$ that is the composition of the left adjoint of the inclusion $\underline{\mathcal{C}}_{\leqslant\left(\underline{\mu}^{\prime}, \nu_{n}\right)} \hookrightarrow \underline{\mathcal{C}}$ and the projection $\underline{\mathcal{C}}_{\leqslant\left(\underline{\boldsymbol{\mu}}^{\prime}, \nu_{n}\right)} \rightarrow \mathcal{C}_{\left(\underline{\boldsymbol{\mu}}^{\prime}, \nu_{n}\right)}$. The functor $\rho_{\underline{\boldsymbol{\mu}}^{\prime},+}$ is exact on $\mathcal{C}^{\bar{\Delta}}$. Therefore all functors in (4.3) are exact.

From the weight considerations, all functors in the exact sequence are 0 unless $\underline{\boldsymbol{\mu}}^{\prime}=\underline{\boldsymbol{\mu}}-\alpha_{i}^{\ell}$ for some $\ell=1, \ldots, n-1$. So consider $\underline{\boldsymbol{\mu}}^{\prime}$ of this form. We claim that the rightmost functor is $\mathrm{id}^{\bigotimes \ell-1} \bigotimes_{\ell} E_{i} \otimes \mathrm{id}^{\bigotimes n-\ell-2} \bigotimes_{n} E_{i}^{(r)}$. Indeed, since $\mathcal{E}: \underline{\mathcal{C}}^{-} \rightarrow \underline{\mathcal{C}}^{+}$is an equivalence of standardly stratified categories, we see that the right-most functor equals $\mathcal{E} \circ \rho_{\underline{\boldsymbol{\mu}}^{\prime},-} \circ F_{i}^{!} \circ \Delta_{\underline{\mu},-}$. But $\rho_{\underline{\boldsymbol{\mu}}^{\prime},-} \circ F_{i}^{!} \circ \Delta_{\underline{\boldsymbol{\mu}}}=\rho_{\underline{\boldsymbol{\mu}}^{\prime},-} \circ E_{i} \circ \Delta_{\underline{\boldsymbol{\mu}}}=\mathrm{id}^{\ell-1} \nabla_{\ell} E_{i} \otimes_{\mathrm{id}^{n-\ell-1}}$. Our claim follows since the equivalence $\underline{\mathcal{C}}_{\underline{\boldsymbol{\mu}}^{\prime},-}^{-} \rightarrow \underline{\mathcal{C}}_{\underline{\mu}^{\prime},+}^{+}$is id ${ }^{\bigotimes n-1} \bigotimes_{n} E_{i}^{(\bar{r})}$. Therefore the right functor maps a simple object to an object with simple head. 
Also the middle functor in (4.3) is the sum of $r+1$ copies of the left-most functor. Apply the functors in (4.3) to a simple object $N$. The object on the right has simple head. It follows that the set of embeddings $\mathbb{K} \hookrightarrow \mathbb{K}^{r+1}$ such that the composition in (4.3) is surjective on $N$ is a complement to a hyperplane in $\mathbb{P}^{r}$. The number of simples in $\mathcal{C}_{\boldsymbol{\mu},-}$ with given $|\underline{\boldsymbol{\mu}}|$ is finite so we have an open subset of $\mathbb{P}^{r}$ such that the composition is surjective if we choose our embedding $\mathbb{K} \hookrightarrow \mathbb{K}^{r+1}$ in this subset. But using condition (TPC3) in the definition of a tensor product categorification one sees that

$$
\left[\rho_{\underline{\boldsymbol{\mu}}^{\prime},+} E_{i}^{r+1} \Delta_{\underline{\boldsymbol{\mu}},-}(N)\right]=(r+1) !\left[\mathrm{id}^{\bigotimes \ell-1} \otimes_{\ell} E_{i} \otimes \mathrm{id}^{\bigotimes n-\ell-2} \otimes_{n} E_{i}^{(r)}(N)\right] .
$$

It follows that the classes of $\rho_{\underline{\boldsymbol{\mu}}^{\prime},+} E_{i}^{(r+1)} \Delta_{\underline{\boldsymbol{\mu}},-}(N)$ and $\rho_{\underline{\boldsymbol{\mu}}^{\prime},+} E_{i}^{(r)} \circ F_{i}^{!} \Delta_{\underline{\boldsymbol{\mu}},-}(N)$ in the Grothendieck group coincide. So any epimorphism between the two objects has to be an isomorphism. This completes the proof.

Using the identification $\mathcal{E}: \underline{\mathcal{C}}^{-} \hookrightarrow \underline{\mathcal{C}}^{+}$, we can transfer $\underline{E}_{i}$ to $\underline{\mathcal{C}}^{+}$. Let $\underline{F}_{i}: \underline{\mathcal{C}}^{+} \rightarrow \underline{\mathcal{C}}^{+}$denote the functor induced by $F_{i}$. Thanks to Lemma 4.7, we see that, being both left and right adjoint to $\underline{F}_{i}$, the functor $\underline{E}_{i}$ preserves the subcategories $\left(\underline{\mathcal{C}}^{ \pm}\right)^{\bar{\Delta}},\left(\underline{\mathcal{C}}^{ \pm}\right)^{\Delta},\left(\underline{\mathcal{C}}^{ \pm}\right)^{\bar{\nabla}},\left(\underline{\mathcal{C}}^{ \pm}\right)^{\nabla}$.

Lemma 4.9. Under the embedding $\pi^{!}:\left(\underline{\mathcal{C}}^{+}\right)^{\bar{\Delta}} \hookrightarrow \mathcal{C}^{\bar{\Delta}}$, we have an isomorphism of functors $\pi^{!} \circ \underline{E}_{i} \cong E_{i} \circ \pi^{!}$. The induced isomorphism $\pi^{!} \circ \underline{E}_{i}^{n} \cong E_{i}^{n} \circ \pi^{!}$intertwines the $R$ actions on these functors.

Proof. It follows from condition (TPC3) that $E_{i}$ preserves $\pi^{!}\left(\underline{\mathcal{C}}^{+}\right)^{\bar{\Delta}}$, so it induces a functor $E_{i}^{\prime}:\left(\underline{\mathcal{C}}^{+}\right)^{\bar{\Delta}} \rightarrow\left(\underline{\mathcal{C}}^{+}\right)^{\bar{\Delta}}$ such that $\pi^{!} \circ E_{i}^{\prime} \cong E_{i} \circ \pi^{!}$. In fact, this functor can be described as $E_{i}^{\prime} \cong \pi \circ E_{i} \circ \pi^{!}$. The right adjoint of this functor is $\pi \circ F_{i} \circ \pi^{*} \cong \underline{F}_{i}$. Taking the left adjoint of this isomorphism, we obtain an isomorphism $E_{i}^{\prime} \cong \underline{E}_{i}$. Since the action of $R$ on $\underline{F}^{n}$ is by definition induced by that on $\pi \circ F^{n} \circ \pi^{*}$, the adjoint isomorphism also intertwines these actions on $\pi \circ E^{n} \circ \pi^{!}$and $\underline{E}^{n}$.

\subsection{Checking conditions.}

Theorem 4.10. The functors $\underline{E}_{i}$ and $\underline{F}_{i}$ give rise to a categorical action on $\underline{\mathcal{C}}^{+}$inducing the tensor product action on $\left[\underline{\mathcal{C}}^{+}\right] \cong V_{1} \otimes \cdots \otimes V_{n-1}$.

Proof. Here we apply [Rou, 5.27]:

- We have already checked that $\underline{E}_{i}$ and $\underline{F}_{i}$ are adjoint.

- The functors $\underline{E}^{k}$ inherit a KLR action from $\mathcal{C}$.

- It's clear that these functors change weights in the correct way.

Thus, we need only check that the linear maps $\left[\underline{E}_{i}\right]$ and $\left[\underline{F}_{i}\right]$ induce an integrable action of $\mathfrak{g}$ on $\left[\underline{\mathcal{C}}^{+}\right] \cong V_{1} \otimes \cdots \otimes V_{n-1}$. Of course, we can easily check that they act with the usual tensor product action; this follows for $\left[E_{i}\right]$ by Lemma 4.9 , and for the $\left[F_{i}\right]$, one simply notes that $\pi^{!}\left(\underline{F}_{i} \Delta_{+}(\underline{\boldsymbol{\lambda}})\right)$ is the kernel of the natural map $F_{i} \Delta(\underline{\boldsymbol{\lambda}}) \rightarrow \Delta\left({ }_{n} F_{i} Q(\underline{\boldsymbol{\lambda}})\right)$; this kernel is, of course, filtered by $\Delta\left({ }_{j} F_{i} Q(\underline{\boldsymbol{\lambda}})\right)=\pi^{!} \Delta\left({ }_{j} \underline{F}_{i} Q(\underline{\boldsymbol{\lambda}})\right)$. This precisely shows that

$$
\left[\underline{F}_{i}\right]\left(v_{1} \otimes \cdots v_{n-1}\right)=\sum_{j=1}^{n-1} v_{1} \otimes \cdots \otimes\left[\underline{F}_{i}\right] v_{j} \otimes \cdots \otimes v_{n-1}
$$

Thus, we are done.

Corollary 4.11. With its induced categorical action, $\underline{\mathcal{C}}^{+}$is a tensor product categorification of $V_{1} \otimes \cdots \otimes V_{n-1}$. 
Proof. Conditions (TPC1-2) are straightforward from the construction. Condition (TPC3) for $F_{i}$ follows directly from the construction of $F_{i}$ as an induced functor. Condition (TPC3) for $E_{i}$ follows from Lemma 4.9.

There is also a "dual" splitting that will be used below. While our original splitting is designed to be compatible with projectives, the dual one is rather compatible with tiltings.

The category which is splitting off is the subcategory $\overline{\mathcal{C}}^{-} \subset \mathcal{C}$ spanned by all simples $L(\lambda)$ with $\varrho(\lambda)=\left(\nu_{1}, \ldots\right)$. The set $\left(\nu_{1}, \ldots\right)$ is a poset ideal and so $\overline{\mathcal{C}}^{-} \subset \mathcal{C}$ is a standardly stratified subcategory. This subcategory is stable with respect to the functors $\underline{E}_{i}:=E_{i}$ but not stable with respect to $F_{i}$. However one can truncate the functors $F_{i}$ getting the endofunctors $\underline{F}_{i}$ completely analogously to the above (first constructing the equivalence $\mathcal{F}$ of $\overline{\mathcal{C}}^{-}$with a suitable subquotient of $\mathcal{C}$ and then showing that $\underline{E}_{i}^{!} \cong \underline{E}_{i}^{*}$ by analogy with Lemma 4.8, we remark that we do not need an analog of Lemma 4.9). With these functors, $\overline{\mathcal{C}}_{-}$becomes a tensor product categorification of $V_{2} \otimes \ldots \otimes V_{n}$.

Remark 4.12. Another way to see this categorification on $\overline{\mathcal{C}}^{-}$is to conjugate our primary construction by Ringel duality: we have $\overline{\mathcal{C}}^{-}={ }^{\vee}\left(\underline{\mathcal{C}}^{\vee+}\right)$, where we write $\vee_{\bullet}:=\left[(\bullet \text { opp })^{\vee}\right]^{\text {opp }}$. The categorification functors $E_{i}$ on ${ }^{\vee}\left(\underline{\mathcal{C}}^{\vee+}\right)$ coincide with the restrictions of $E_{i}$ to $\overline{\mathcal{C}}^{-}$; this can be seen from Lemma 4.9 (applied to $\mathcal{C}^{\vee o p p}$ ).

\section{Double CENTRAlizer PROPERTY}

5.1. Statement. Let $\mathcal{C}$ be a tensor product categorification of $V_{1} \otimes \cdots \otimes V_{n}$. As before let $\underline{\boldsymbol{\nu}}=\left(\nu_{1}, \ldots, \nu_{n}\right)$ be the sequence of highest weights of $V_{1}, \ldots, V_{n}$, and we write $|\nu|$ for $\sum_{i=1}^{n} \nu_{n}$. We consider the projectives in $\mathcal{C}$ that are direct summands of $F^{N} \mathbb{V}$ for $N \in \mathbb{Z}_{\geqslant 0}$, where $\mathbb{V}$ was defined in (TPC2). The corresponding quotient functor $\pi_{t o p}$ kills all simples $L(\lambda)$ such that $|\underline{\boldsymbol{\nu}}-\varrho(\lambda)|$ is the sum of $k$ simple roots but $E^{k} L(\lambda)=0$. The quotient category categorifies the Cartan irreducible component of $V_{1} \otimes \cdots \otimes V_{n}$ (=the only irreducible component with highest weight $|\underline{\boldsymbol{\nu}}|)$; we denote it by $\mathcal{C}_{\text {top }}$. The category $\mathcal{C}_{\text {top }}$ has an induced categorical action which makes $\pi_{\text {top }}$ strongly equivariant. By Proposition $3.2, \mathcal{C}_{\text {top }}$ is strongly equivariantly equivalent to the representations of a cyclotomic quotient of the KLR algebra $R^{|\underline{\nu}|}$-mod. As shown in the proof of that theorem, this functor can be identified with $M \mapsto \operatorname{Hom}\left(\mathbb{V}, E^{k} M\right)=$ $\operatorname{Hom}\left(F^{k} \mathbb{V}, M\right)$; this has a canonical action of the KLR algebra which factors through the cyclotomic quotient $R^{|\nu|}$.

We can canonically identify $\mathcal{C}_{\text {top }}$-proj $\cong \mathcal{C}_{\text {top }}$-inj with the subcategory of $\mathcal{C}$ additively generated by the objects $F^{N} \mathbb{V}$.

Here is the main result of this section.

Theorem 5.1. The functor $\pi_{\text {top }}$ is fully faithful on projectives.

Our approach closely follows Soergel's, [Soe90], to prove Struktursatz. An essential prerequisite for this proof is to check that every simple appearing in the socle of an object from $\mathcal{C}^{\bar{\Delta}}$ survives under $\pi_{\text {top }}$.

\subsection{Socles of standard objects.}

Proposition 5.2. Pick $\underline{\boldsymbol{\mu}}$ such that $|\underline{\boldsymbol{\nu}}-\underline{\boldsymbol{\mu}}|$ is the sum of $k$ simple roots. If a simple $L$ appears in the socle of $\Delta_{\underline{\mu}}(N)$ with $N \in \mathcal{C}_{\underline{\mu}}$, then $E^{k} L \neq 0$.

Proof. The claim is vacuous when $n=1$. So in the proof we can assume that the claim is proved for all tensor product categorifications of products with $n-1$ factors, in particular, 
for $\underline{\mathcal{C}}^{-} \subset \mathcal{C}$. Since the subcategory $\underline{\mathcal{C}}^{-}$is closed under $E$, this establishes our claim when $\mu_{1}=\nu_{1}$. From now on we may assume that $\mu_{1}<\nu_{1}$.

Now we prove our claim by induction on the number $\ell$ of simple roots in the decomposition of $\nu_{1}-\mu_{1}$. We may assume that $N$ is simple, $N=N_{1} \otimes \cdots \otimes N_{n}$. Since $\mu_{1}<\nu_{1}$, we see that $N_{1}$ occurs in the socle (equivalently, top) of $F_{i} N_{1}^{\prime}$ for some $i$ and some simple $N_{1}^{\prime} \in \mathcal{C}_{\mu_{1}+\alpha_{i}}^{1}$. Set $N^{\prime}=N_{1}^{\prime} \otimes N_{2} \otimes \ldots \otimes N$ and consider the object $F_{i} \Delta_{\underline{\mu}+\alpha_{i}^{1}}\left(N^{\prime}\right)$. According to condition (TPC3), this object has a filtration with subobject $\Delta_{\underline{\mu}}\left({ }_{1} \bar{F}_{i} N^{\prime}\right)$. This induces an injection $\Delta_{\underline{\mu}}(N) \hookrightarrow F_{i} \Delta_{\underline{\mu}+\alpha_{i}^{1}}\left(N^{\prime}\right)$, and thus an injection

$$
\operatorname{Hom}\left(L, \Delta_{\underline{\mu}}(N)\right) \hookrightarrow \operatorname{Hom}\left(L, F_{i} \Delta_{\underline{\mu}+\alpha_{i}^{1}}\left(N^{\prime}\right)\right)=\operatorname{Hom}\left(E_{i} L, \Delta_{\underline{\boldsymbol{\mu}}+\alpha_{i}^{1}}\left(N^{\prime}\right)\right) .
$$

Thus, if the former space is nonzero, the latter is as well. Any non-zero map $E_{i} L \rightarrow$ $\Delta_{\underline{\mu}+\alpha_{i}^{1}}\left(N^{\prime}\right)$ induces an injection of a simple composition factor $L^{\prime}$ of $E_{i} L$ into the socle of $\Delta_{\mu+\alpha_{i}^{1}}\left(N^{\prime}\right)$. By the inductive assumption, $E^{k-1} L^{\prime} \neq 0$ and hence $E^{k} L \neq 0$ by the exactness of $E$.

Lemma 5.3. For any $M \in \mathcal{C}^{\Delta}$, we have an injection $M \hookrightarrow P$ with $P \in \mathcal{C}_{\text {top }}$-proj. Moreover, if $M \in \mathcal{C}$-proj, then we can choose this map so that $P / M \in \mathcal{C}^{\Delta}$.

Proof. By Proposition 5.2, the injective hull of $M \in \mathcal{C}^{\Delta}$ lies in $\mathcal{C}_{\text {top }}$-inj $=\mathcal{C}_{\text {top }}$-proj, which shows that the desired injection exists. For the remainder of the proof, we assume that $M \in \mathcal{C}$-proj.

We prove this by induction on $k$, where $k$ has the same meaning as in Proposition 5.2. If $k=0$, then this is trivial. Now, fix $k$, and assume the statement holds for $k-1$. Then for any $M \in \mathcal{C}$-proj, we have that $E M \in \mathcal{C}$-proj, so by induction, we have a map $E M \hookrightarrow P^{\prime}$ for $P^{\prime} \in \mathcal{C}_{\text {top }}$-proj and $P^{\prime} / E M \in \mathcal{C}^{\Delta}$. This gives rise to an embedding $F E M \hookrightarrow F P^{\prime}$ and we have $F P^{\prime} / F E M=F\left(P^{\prime} / E M\right) \in \mathcal{C}^{\Delta}$ by (TPC3). So it remains to show that there is an embedding $M \hookrightarrow F E M$ with $F E M / M \in \mathcal{C}^{\Delta}$. We take the morphism $M \rightarrow F E M$ obtained from the identity morphism $E M \rightarrow E M$ by adjunction. The induced morphism $E M \rightarrow E F E M$ is an embedding. Since there is no simple in the socle of $M$ killed by $E$, we deduce that the morphism $M \rightarrow F E M$ is an embedding.

By Lemma 2.4 (2), it remains to check that $\operatorname{Ext}^{1}(F E M / M, \bar{\nabla}(\underline{\boldsymbol{\lambda}}))=0$ for all $\underline{\boldsymbol{\lambda}}$. From the usual long exact sequence, this is equivalent to the surjectivity of the induced map

$$
\operatorname{Hom}(F E M, \bar{\nabla}(\underline{\boldsymbol{\lambda}})) \rightarrow \operatorname{Hom}(M, \bar{\nabla}(\underline{\boldsymbol{\lambda}}))
$$

for the unit $M \rightarrow F E M$ of the adjunction $(E, F)$; by the biadjunction of $F$ and $E$, this is in turn equivalent to the surjectivity of the map

$$
\operatorname{Hom}(M, F E \bar{\nabla}(\underline{\boldsymbol{\lambda}})) \rightarrow \operatorname{Hom}(M, \bar{\nabla}(\underline{\boldsymbol{\lambda}}))
$$

induced by the surjective counit map $F E \bar{\nabla}(\underline{\boldsymbol{\lambda}}) \rightarrow \bar{\nabla}(\underline{\boldsymbol{\lambda}})$ for the adjunction $(F, E)$. Of course, this is just the universal property of projectives.

Proof of Theorem 5.1. It is enough to check that $\operatorname{Hom}_{\mathcal{C}}\left(M, M^{\prime}\right) \stackrel{\sim}{\rightarrow} \operatorname{Hom}_{\mathcal{C}^{t o p}}\left(\pi_{\text {top }} M, \pi_{\text {top }} M^{\prime}\right)$ when $M^{\prime} \in \mathcal{C}$-proj. By Lemma [5.3, we can write $M^{\prime}$ as the cokernel of a map

$$
0 \rightarrow M^{\prime} \rightarrow P_{1} \rightarrow P_{2}
$$

for $P_{1}, P_{2} \in \mathcal{C}_{\text {top }}$-proj. We thus have a short exact sequence

$$
0 \rightarrow \pi_{\text {top }}\left(M^{\prime}\right) \rightarrow \pi_{\text {top }}\left(P_{1}\right) \rightarrow \pi_{\text {top }}\left(P_{2}\right),
$$


with the latter two terms injective modules. Essentially by definition, we have that $\operatorname{Hom}_{\mathcal{C}}\left(M, P_{i}\right) \cong$ $\operatorname{Hom}_{\mathcal{C}^{\text {top }}}\left(\pi_{\text {top }} M, \pi_{\text {top }} P_{i}\right)$ for any module $M$. Thus, we have the diagram with exact rows

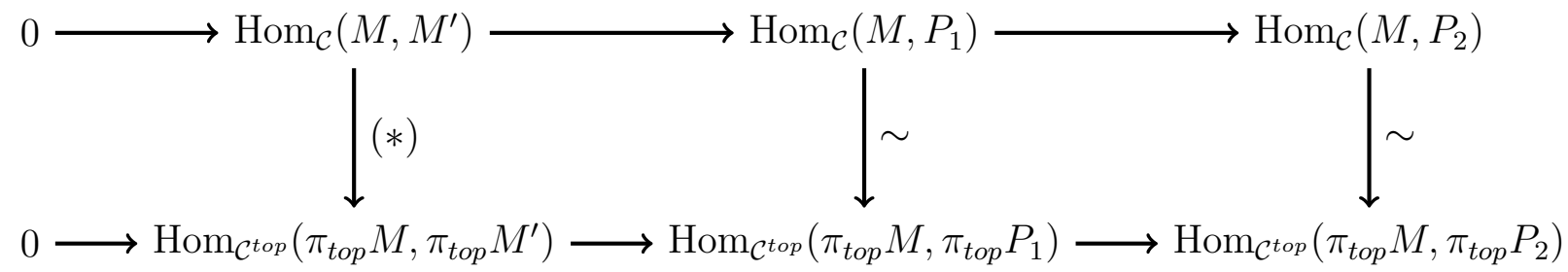

By the 5-lemma, the map marked $(*)$ is an isomorphism.

\section{Proof of the uniqueness theorem}

6.1. Main result. We intend to give a classification of all tensor product categorifications.

Theorem 6.1. Let $\mathcal{C}$ be a tensor product categorification of $V_{1} \otimes \cdots \otimes V_{n}$. Then we have a strongly equivariant equivalence $\mathcal{C} \cong \mathcal{C}(\underline{\boldsymbol{\nu}})$ of standardly stratified categories that preserves the labels of simples.

Consider the categorification $\underline{\mathcal{C}}^{+}$; since the highest weight of this categorification is $|\nu|-\nu_{n}$, we have a functor $\underline{\pi}_{\text {top }}$ from $\underline{\mathcal{C}}^{+}$to the category $R^{|\nu|-\nu_{n}}$-mod. We wish to compare this to the functor $\pi_{t o p}$.

Lemma 6.2. We have a commutative diagram

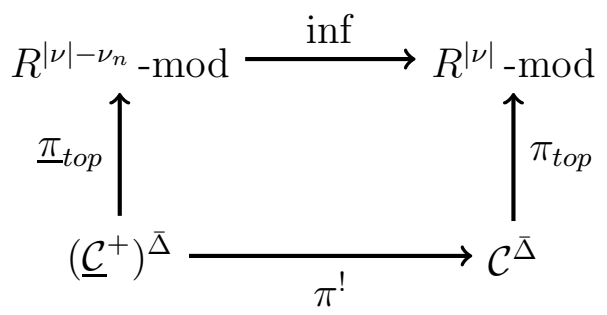

Proof. By Lemma 4.9, the vector spaces $E^{k} \pi^{!}(M)$ and $\underline{E}^{k}(M)$ are naturally isomorphic as $R$-modules. Thus, if we think of $E^{k} \pi^{!}(M)$ as an $R^{|\underline{\underline{\nu}}|}$-module, it is simply obtained by considering $\underline{E}^{k}(M)$ as an $R^{|\underline{\boldsymbol{\nu}}|-\nu_{n}}$-module and pulling back.

Proof of Theorem 6.1. Let $\pi_{1}, \pi_{2}$ denote the quotient functors $\mathcal{C} \rightarrow \underline{\mathcal{C}}^{+}$and $\mathcal{C}\left(\nu_{1}, \ldots, \nu_{n}\right) \rightarrow$ $\mathcal{C}\left(\nu_{1}, \ldots, \nu_{n-1}\right)$. Further let $\pi_{\text {top }}^{1}, \pi_{\text {top }}^{2}$ be the quotient functors from $\mathcal{C}$ and $\mathcal{C}\left(\nu_{1}, \ldots, \nu_{n}\right)$ to $R^{|\underline{\nu}|}$-mod and $\underline{\pi}_{\text {top }}^{1}, \underline{\pi}_{\text {top }}^{2}$ the quotient functors from $\underline{\mathcal{C}}^{+}, \mathcal{C}\left(\nu_{1}, \ldots, \nu_{n-1}\right)$ to $R^{|\underline{\underline{\nu}}|-\nu_{n}} \bmod$. Below we will sometimes write $\mathcal{C}_{1}$ for $\mathcal{C}$, and $\mathcal{C}_{2}$ for $\mathcal{C}\left(\nu_{1}, \ldots, \nu_{n}\right)$ (and also $\underline{\mathcal{C}}_{1}^{+}$for $\underline{\mathcal{C}}_{+}$and $\underline{\mathcal{C}}_{2}^{+}$ for $\left.\mathcal{C}\left(\nu_{1}, \ldots, \nu_{n-1}\right)\right)$.

First, note that it suffices to check this theorem on the categories of projective objects in each category. By Theorem [5.1, we can strongly equivariantly identify $\mathcal{C}$-proj and $\mathcal{C}\left(\nu_{1}, \ldots, \nu_{n}\right)$-proj with their images under $\pi_{\text {top }}^{i}$. Thus, the desired equivalence would follow from showing that $\left\{\pi_{\text {top }}^{1} P^{1}(\underline{\boldsymbol{\lambda}})\right\}=\left\{\pi_{\text {top }}^{2} P^{2}(\underline{\boldsymbol{\lambda}})\right\}$, where $P^{1}(\underline{\boldsymbol{\lambda}})$ and $P^{2}(\underline{\boldsymbol{\lambda}})$ are the indecomposable projectives corresponding to $\lambda$ in $\mathcal{C}$ and $\mathcal{C}\left(\nu_{1}, \ldots, \nu_{n}\right)$. To show that the equivalence is of stratified categories and preserves the labels of simples, it is enough to check that $\pi_{\text {top }}^{1} P^{1}(\underline{\boldsymbol{\lambda}})=\pi_{\text {top }}^{2} P^{2}(\underline{\boldsymbol{\lambda}})$ (indeed, the only additional structure on a standardly stratified category is a pre-order on the set of simples so if an equivalence preserves the labels, then it will automatically intertwine the standardly stratified structures). 
Our proof proceeds by induction. If $n=1$, then $\mathcal{C} \cong \operatorname{gr} \mathcal{C}$, and the conclusion follows from Proposition 3.2 .

For arbitrary $n$, we can conclude by the inductive hypothesis that $\underline{\mathcal{C}}^{+} \cong \mathcal{C}\left(\nu_{1}, \ldots, \nu_{n-1}\right)$ and the equivalence preserves the labels of the simples. Such an equivalence automatically intertwines the functors $\underline{\pi}_{\text {top }}^{1}$ and $\underline{\pi}_{\text {top }}^{2}$. Thus, by Lemma $\left[6.2, \pi_{\text {top }}^{1} \circ \pi_{1}^{!}\left(P_{+}^{1}(\underline{\boldsymbol{\lambda}})\right)=\pi_{\text {top }}^{2} \circ\right.$ $\pi_{2}^{!}\left(P_{+}^{2}(\underline{\boldsymbol{\lambda}})\right)$ for all labels $\underline{\boldsymbol{\lambda}}$ in $\underline{\mathcal{C}}^{+} \cong \mathcal{C}\left(\nu_{1}, \ldots, \nu_{n-1}\right)$. Since $\pi_{i}^{!} P_{+}^{i}(\underline{\boldsymbol{\lambda}})=P^{i}(\underline{\boldsymbol{\lambda}})$, we see that the objects $\pi_{\text {top }}^{i} P^{i}(\underline{\boldsymbol{\lambda}})$ coincides when $\underline{\boldsymbol{\lambda}}=\left(\lambda_{1}, \ldots, \lambda_{n}\right)$ with $\varrho^{n}\left(\lambda_{n}\right)=\nu_{n}$.

We claim that any projective $P^{i}\left(\underline{\boldsymbol{\lambda}}^{\prime}\right)$ appears as a summand in $F^{N} P^{i}(\underline{\boldsymbol{\lambda}})$. Indeed, the classes of projectives $P^{i}(\underline{\boldsymbol{\lambda}})$ with $\varrho^{n}\left(\lambda_{n}\right)=\nu_{n}$ are a basis in $V_{1} \otimes \ldots \otimes V_{n-1} \otimes v_{\nu_{n}} \subset V_{1} \otimes \ldots \otimes V_{n}$. Since $V_{1} \otimes \ldots \otimes V_{n-1} \otimes v_{\nu_{n}}$ generates the $U\left(\mathfrak{n}^{-}\right)$-module $V_{1} \otimes \ldots \otimes V_{n}$, we see that the classes of summands of $F^{N} P^{i}(\underline{\boldsymbol{\lambda}})$ (for all $N$ and $\underline{\boldsymbol{\lambda}}$ ) generate $V_{1} \otimes \ldots \otimes V_{n}$, so there can be no other projectives.

So any $\pi_{\text {top }}^{i} P^{i}\left(\underline{\boldsymbol{\lambda}}^{\prime}\right)$ is an indecomposable (thanks to Theorem 5.1$)$ summand of $F^{N} \pi_{\text {top }}^{i} P^{i}(\underline{\boldsymbol{\lambda}})$ with $\varrho^{n}\left(\lambda_{n}\right)=\nu_{n}$. It follows that the sets $\left\{\pi_{\text {top }}^{i} P^{i}(\lambda)\right\}$ coincide, which proves the existence of a strongly equivariant equivalence $\beta: \mathcal{C} \rightarrow \mathcal{C}\left(\nu_{1}, \ldots, \nu_{n}\right)$.

Now, we need only check that the labels match. Let us choose a sequence of nodes $i_{1}, i_{2}, \ldots$ as in Section 3.3, and order infinite sequences of non-negative integers almost all of which are 0 by:

- If $\sum a_{i}<\sum b_{i}$, then $\mathbf{a}=\left(a_{1}, \ldots\right)>\mathbf{b}=\left(b_{1}, \ldots\right)$.

- If $\sum a_{i}=\sum b_{i}$, then we use lexicographic order.

For each indecomposable projective object $P^{i}(\underline{\boldsymbol{\lambda}})$, where $i=1,2$, there is a unique word $\mathbf{a}=$ $\left(a_{1}, \ldots\right)$ maximal in this order for which $P^{i}(\underline{\boldsymbol{\lambda}})$ appears in $F_{i_{1}}^{a_{1}} F_{i_{2}}^{a_{2}} \cdots Q$ for $Q$ projective in $\mathcal{C}_{+}^{i}$. This is equivalent to a being maximal so that $Q$ has a non-zero map to $\cdots E_{i_{2}}^{a_{2}} E_{i_{1}}^{a_{1}} L^{i}(\underline{\boldsymbol{\lambda}})$. In particular, the standard cover of some composition factor of $\cdots E_{i_{2}}^{a_{2}} E_{i_{1}}^{a_{1}} L^{i}(\underline{\boldsymbol{\lambda}})$ must have a label whose $n$th component is $v_{n}$, the only indecomposable object in $\mathcal{C}_{\nu_{n}}^{n}$. We call such a label a plus-label. Note that a simple with plus-label can only occur as a composition factor of a standard with plus-label.

Let $\mathbf{b}$ be the string parameterization, see Section 3.3 , of $\lambda_{n}$. Assume $\mathbf{a}$ is larger than $\mathbf{b}$ in the order above.

Now, consider $\cdots E_{i_{2}}^{a_{2}} E_{i_{1}}^{a_{1}} L^{i}(\underline{\boldsymbol{\lambda}})$. This is a quotient of $\cdots E_{i_{2}}^{a_{2}} E_{i_{1}}^{a_{1}} \bar{\Delta}^{i}(\underline{\boldsymbol{\lambda}})$; this module has a canonical filtration by proper standard modules. If $\mathbf{a}$ has smaller sum than $\mathbf{b}$, then none of these standards has plus-label, since even the successive quotient where we only use E's from the last component cannot have high enough weight in the last label. On the other hand, if a has the same sum, but is higher in lexicographic order, then the component of the standard filtration where we apply all E's in the last component will have the correct weight, but be trivial by the definition of string parametrization. Thus, the word a mentioned above must be no greater in our order than $\mathbf{b}$.

On the other hand, we claim that the object $\cdots E_{i_{2}}^{b_{2}} E_{i_{1}}^{b_{1}} L^{i}(\underline{\boldsymbol{\lambda}})$ contains the simple with label $\underline{\boldsymbol{\lambda}}^{+}$matching $\underline{\boldsymbol{\lambda}}$ except with $v_{n}$ in the last component as a composition factor. Indeed, by (TPC3), $\bar{\Delta}^{i}\left(\underline{\boldsymbol{\lambda}}^{+}\right)$is a composition factor of $\cdots E_{i_{2}}^{b_{2}} E_{i_{1}}^{b_{1}} \bar{\Delta}^{i}(\underline{\boldsymbol{\lambda}})$ and the label $\underline{\boldsymbol{\lambda}}^{+}$is the largest among the labels of the composition factors. If $L^{i}\left(\underline{\boldsymbol{\lambda}}^{+}\right)$appears in the kernel of the projection $\cdots E_{i_{2}}^{b_{2}} E_{i_{1}}^{b_{1}} \bar{\Delta}^{i}(\underline{\boldsymbol{\lambda}}) \rightarrow \cdots E_{i_{2}}^{b_{2}} E_{i_{1}}^{b_{1}} L^{i}(\underline{\boldsymbol{\lambda}})$, then it also appears in $\cdots E_{i_{2}}^{b_{2}} E_{i_{1}}^{b_{1}} \bar{\Delta}^{i}\left(\underline{\boldsymbol{\lambda}^{\prime}}\right)$ for some $\underline{\boldsymbol{\lambda}}^{\prime}<\underline{\boldsymbol{\lambda}}$. This is impossible if $\lambda_{n}^{\prime}<\lambda_{n}$. Furthermore, if $\lambda_{n}^{\prime}=\lambda_{n}$, then the largest label of a composition factor in $\cdots E_{i_{2}}^{b_{2}} E_{i_{1}}^{b_{1}} \bar{\Delta}^{i}\left(\underline{\boldsymbol{\lambda}}^{\prime}\right)$ is $\underline{\boldsymbol{\lambda}}^{\prime+}<\underline{\boldsymbol{\lambda}}^{+}$. This contradiction shows the claim in the beginning of the paragraph that, in turn, implies $a_{i}=b_{i}$ for all $i$. 
Note that all other proper standards appearing in $\cdots E_{i_{2}}^{a_{2}} E_{i_{1}}^{a_{1}} \bar{\Delta}^{i}(\underline{\boldsymbol{\lambda}})$ do not have plus-labels, and thus have no simple composition factors with plus-labels. Thus, if any other simple with a plus label occurs as a composition factor $\cdots E_{i_{2}}^{b_{2}} E_{i_{1}}^{b_{1}} L^{i}(\underline{\boldsymbol{\lambda}})$, then is must a composition factor of $\bar{\Delta}\left(\underline{\boldsymbol{\lambda}}^{+}\right)$. Thus, every other plus-label attached to a simple in $\cdots E_{i_{2}}^{b_{2}} E_{i_{1}}^{b_{1}} L^{i}(\underline{\boldsymbol{\lambda}})$ must be $<\underline{\boldsymbol{\lambda}}^{+}$.

This shows the uniqueness of labels: the word $\mathbf{a}$ is determined by the definition of the action and the category $\mathcal{C}^{+}$, and so must match for $P$ and $\beta(P)$; this shows that the last term of the labels match, and the label $\underline{\boldsymbol{\lambda}}^{+}$is distinguished as the maximal plus-label for a composition factor in $\cdots E_{i_{2}}^{a_{2}} E_{i_{1}}^{a_{1}} L^{i}(\underline{\boldsymbol{\lambda}})$. Since we already know that the labels match for $\mathcal{C}^{+}$ by induction, this establishes the general case.

6.2. Consequences. This theorem shows that many structures on tensor product categorifications thus come for free:

Corollary 6.3. If the polynomials $Q_{i j}(u, v)$ are chosen to be homogeneous, then any tensor product categorification has a unique graded lift (given by graded modules of $T^{\boldsymbol{\nu}}$ ) which carries a homogeneous action of $\mathfrak{A}$; that is, between any two such lifts, there is a strongly equivariant graded lift of the identity functor which is unique up to unique isomorphism.

Proof. The existence of this lift is clear, so we turn to its uniqueness. The natural map $R_{k} \rightarrow \operatorname{End}\left(F^{k} \mathbb{V}\right)$ is surjective with homogeneous kernel, and thus induces a grading on the latter space. Furthermore, every indecomposable projective has an injective map into $F^{k} \mathbb{V}^{\oplus p}$ for some $p$ (its injective hull is a summand of this module). We call a projective submodule $P \subset F^{k} \mathbb{V}^{\oplus p}$ homogeneous if the left ideal of $\operatorname{End}\left(F^{k} \mathbb{V}^{\oplus p}\right)$ consisting of endomorphisms whose image lies in $P$ is homogeneous for this grading. Note that this module coincides with $\operatorname{Hom}\left(F^{k} \mathbb{V}^{\oplus p}, P\right)$ and thus grades this space. By the double centralizer property, this induces a grading on the Hom space between any two homogeneous projective submodules of $F^{k} \mathbb{V}^{\oplus p}$.

The category of homogeneous projective submodules of $F^{k} \mathbb{V}^{\oplus p}$ is thus a graded lift of the category of projectives in $\mathcal{C}(\underline{\boldsymbol{\nu}})$, which only depends on the choice of grading on $R_{k}$. Any other graded lift has a canonical functor from its category of homogeneous projectives to this lift induced by the functor $\pi_{t o p}$, so this establishes the desired uniqueness.

Corollary 6.4 ([Webb, Thm. B]). The tensor product categorifications for different orderings of the same representations have equivalent derived categories.

As mentioned before, there are different, competing notions of categorical $\mathfrak{g}$-action; the most obvious variation of the definition we have used is the 2-category of Cautis and Lauda, where rather than simply adjoining an inverse of $\rho_{s, \lambda}$, the relation that another morphism is its inverse is imposed. The difference between these definitions is subtle, and the evidence thus far suggests that most interesting actions in the sense of Rouquier can be strengthened to one of these. For example, in our case, we find:

Corollary 6.5 ([Webb, Thm. A]). Any tensor product action can be strengthened to an action of the 2-category of Cautis and Lauda [CL].

Moreover, Theorem 6.1 provides a new and independent proof of [Weba, Corollary 4.7], which establishes an equivalence of $\mathcal{C}$, the sum of blocks in a parabolic category $\mathcal{O}$ mentioned in Section 3.5, and the category $\mathcal{C}\left(\omega_{m_{1}}, \omega_{m_{2}}, \ldots, \omega_{m_{n}}\right)$. It also shows that the subquotient categories $\mathcal{C}_{e}$ from Section 3.5 only depend on the ordered products they categorify and not on the equivalence class $e$. 
These techniques are also useful for analyzing the category $\mathcal{O}$ for a Lie superalgebra $\mathfrak{g l}(m \mid n)$. These applications will be explored further is a forthcoming joint paper of Brundan and the authors [BLW].

\section{CRystals}

7.1. Main result. Recall that we have defined crystal operators $\tilde{e}_{i}, \tilde{f}_{i}$ on the set of simples in a tensor product categorification $\mathcal{C}$ of $V_{1} \otimes \cdots \otimes V_{n}$; this induces a crystal structure on $\Lambda$, which we call the categorical crystal structure to avoid confusion.

Our main result in this section is to give a combinatorial description of this structure; both the result and its proof generalize those from [Losa]. Recall that the set of labels for simples in $\mathcal{C}$ is $\left\{\underline{\boldsymbol{\lambda}}=\left(\lambda_{1}, \ldots, \lambda_{n}\right)\right\}$, where $\lambda_{j}$ is a label of a simple in the categorification $\mathcal{C}^{j}$ of $V_{j}$. Recall that the crystal of $\mathcal{C}^{j}$ is known: it is isomorphic to the crystal of $V_{j}$ by [LV11, §5.1]. Since this crystal is irreducible, this determines the crystal operators, ${ }_{j} \tilde{e}_{i},{ }_{j} \tilde{f}_{i}$, uniquely.

To describe the crystal operators for $\mathcal{C}$, we will need some notation and the notion of the $i$-signature. Let

- $h_{+}^{j}\left(\lambda_{j}\right)$ be the maximum over all integers $k$ such that $\left({ }_{j} E_{i}\right)^{k} L^{j}\left(\lambda_{j}\right) \neq 0$, and

- $h_{-}^{j}\left(\lambda_{j}\right)$ be maximum over all integers $\ell$ such that $\left({ }_{j} F_{i}\right)^{\ell} L^{j}\left(\lambda_{j}\right) \neq 0$.

Definition 7.1. For each index $j$, we have a sign sequence given by concatenating $h_{+}^{j}\left(\lambda_{j}\right)$ many +-signs, followed by $h_{-}^{j}\left(\lambda_{j}\right)$ many --signs. The $i$-signature of $\underline{\boldsymbol{\lambda}}$ is a sequence of + 's and -'s given by concatenating the sequences from each index in turn.

For example, consider the case where $\mathfrak{g}=\mathfrak{s l}_{2}$, and $\nu_{1}=\nu_{2}=\nu_{3}=3$. In this case all $i$-signatures will be of length 9 ; for example $\lambda_{1}$ is the unique crystal elements of weight -1 and $\lambda_{2}, \lambda_{3}$ have weight 1 , then we have three groups: $(++-),(+--),(+--)$, then the $i$-signature is $(++-+--+--)$. Note that outside $\mathfrak{s l}_{2}$, the length of the $i$-signature can differ for labels in the same representation.

We annotate each $i$-signature as follows: if you find a consecutive pair of the form -+ , cross both out, and continue this process, ignoring crossed out symbols, until every pair of uncrossed + and --signs have the + sign to the left. This process is often visualized by replacing + with the open parenthesis symbol ( and - with the close parenthesis ) and ignoring matching parentheses; in [Losa, 2.4], symbols are turned into 0's rather than struck out. This allows us to speak, in any $i$-signature, of crossed and uncrossed symbols. We let $h_{ \pm}(\lambda)$ be the number of uncrossed symbols remaining. In the example above, we strike out the symbols in positions $3,4,6$, and 7 and arrive at $(++\neq \Varangle-\not \nsucc--)$.

Now, we define a second crystal structure on $\Lambda$ using the usual rule for tensor products of crystals $\Lambda=\Lambda_{1} \otimes \cdots \otimes \Lambda_{n}$; we call this the combinatorial crystal structure on $\Lambda$. When we write a $\tilde{e}_{i} \underline{\boldsymbol{\lambda}}$ or $\tilde{f}_{i} \underline{\boldsymbol{\lambda}}$, we will always mean the action in the combinatorial structure, and always write $\tilde{\tilde{e}}_{i} L(\underline{\boldsymbol{\lambda}})$ or $\tilde{f}_{i} L(\underline{\boldsymbol{\lambda}})$ for the categorical structure.

Let us describe the tensor product crystal rule in our language. Assume that the $j$-th group from the left contains the rightmost uncrossed + , and the $j^{\prime}$-th group contains the leftmost uncrossed - ; then we define

$$
\tilde{e}_{i}\left(\lambda_{1}, \ldots, \lambda_{n}\right)=\left(\lambda_{1}, \ldots,{ }_{j} \tilde{e}_{i} \lambda_{j}, \ldots, \lambda_{n}\right) \quad \tilde{f}_{i}\left(\lambda_{1}, \ldots, \lambda_{n}\right)=\left(\lambda_{1}, \ldots,{ }_{j} \tilde{f}_{i} \lambda_{j}, \ldots, \lambda_{n}\right) .
$$

If there is no uncrossed + , we set $\tilde{e}_{i}(\underline{\boldsymbol{\lambda}})=0$, and if there is no uncrossed - , we set $\tilde{f}_{i} \underline{\boldsymbol{\lambda}}=0$. In the example above, $j=1$ and $j^{\prime}=2$. 
We remark that the $i$-signature of $\tilde{e}_{i} \lambda$ is obtained from the $i$-signature of $\lambda$ by switching the rightmost uncrossed + to a - ; the $i$-signature of $\tilde{f}_{i} \lambda$ is obtained by switching the leftmost uncrossed - to a + .

Here is our main theorem concerning the crystal structure of $\mathcal{C}$. It is a partial generalization of the main result of [Losa] and answers a question of the second author, Weba, Conjecture $3.12]$.

Theorem 7.2. The categorical and combinatorial crystal structures coincide. That is

$$
\tilde{e}_{i} L(\lambda)=L\left(\tilde{e}_{i} \lambda\right) \quad \text { and } \quad \tilde{f}_{i} L(\lambda)=L\left(\tilde{f}_{i} \lambda\right) .
$$

Since this can be checked one simple root at a time, we fix $i$ and suppress the subscript $i$ in $E_{i}, \tilde{e}_{i}, \alpha_{i}$, etc. throughout the rest of this section.

There is a weaker version of this claim that is easy to see. For $\underline{\boldsymbol{\lambda}}=\left(\lambda_{1}, \ldots, \lambda_{n}\right)$ we write ${ }_{j} \tilde{e} \underline{\boldsymbol{\lambda}}$ for $\left(\lambda_{1}, \ldots,{ }_{j} \tilde{e} \lambda_{j}, \ldots, \lambda_{n}\right)$. The notation ${ }_{j} \tilde{f} \underline{\boldsymbol{\lambda}}$ has a similar meaning.

Lemma 7.3. We have $\tilde{e} L(\underline{\boldsymbol{\lambda}})=L\left({ }_{j} \tilde{e} \underline{\boldsymbol{\lambda}}\right), \tilde{f} L(\underline{\boldsymbol{\lambda}})=L\left({ }_{j^{\prime}} \tilde{\tilde{f}} \underline{\boldsymbol{\lambda}}\right)$ for some $j, j^{\prime}$.

Proof. We remark that $E \bar{\Delta}(\underline{\boldsymbol{\lambda}}) \rightarrow E L(\underline{\boldsymbol{\lambda}})$ and so $\tilde{e} L(\underline{\boldsymbol{\lambda}})$ lies in the head of $E \bar{\Delta}(\underline{\boldsymbol{\lambda}})$. Thanks to (HWC3), the head of $E \bar{\Delta}(\underline{\boldsymbol{\lambda}})$ lies the direct sum of the heads of the modules $\Delta_{\varrho(\underline{\boldsymbol{\lambda}})+\alpha^{j}}\left({ }_{j} \tilde{e} L_{\varrho(\underline{\boldsymbol{\lambda}})}(\underline{\boldsymbol{\lambda}})\right)$. Since ${ }_{j} \tilde{e} L_{\varrho(\underline{\boldsymbol{\lambda}})}(\underline{\boldsymbol{\lambda}})$, has simple head, equal to $L_{\varrho(\underline{\boldsymbol{\lambda}})+\alpha^{j}}\left({ }_{j} \tilde{\tilde{e}} \underline{\boldsymbol{\lambda}}\right)$, we see that the head of $\Delta_{\varrho(\underline{\boldsymbol{\lambda}})+\alpha^{j}}\left({ }_{j} \tilde{e} L_{\varrho(\underline{\boldsymbol{\lambda}})}(\underline{\boldsymbol{\lambda}})\right)$ equals $L\left({ }_{j} \tilde{\tilde{e}} \underline{\boldsymbol{\lambda}}\right)$. This completes the proof of the first equality of the lemma. The proof of the second one is completely analogous.

7.2. Ext vanishing. As in [Losa], our proof of Theorem 7.2 is based on vanishing of certain Ext's between $\Delta(\underline{\boldsymbol{\mu}})$ 's and $L(\underline{\boldsymbol{\lambda}})$ 's. A result here is a direct generalization of [Losa, Proposition 5.5].

For $k=1, \ldots, n$ and a label $\underline{\boldsymbol{\lambda}}^{\prime}$ define an integer $h_{-, k}\left(\underline{\boldsymbol{\lambda}}^{\prime}\right)$ as the number of -'s in the reduced form of the part of $t$ lying in the groups to the right of and including the $k$ th. In the example we have used before, $h_{-, 1}\left(\underline{\boldsymbol{\lambda}}^{\prime}\right)=h_{-, 2}\left(\underline{\boldsymbol{\lambda}}^{\prime}\right)=3, h_{-, 3}\left(\underline{\boldsymbol{\lambda}}^{\prime}\right)=2$. In general, $h_{-, 1}\left(\underline{\boldsymbol{\lambda}}^{\prime}\right), \ldots, h_{-, n}\left(\underline{\boldsymbol{\lambda}}^{\prime}\right)$ is a (non-strictly) decreasing sequence.

Consider integers $m>0$ such that $F^{m} L(\underline{\boldsymbol{\lambda}})=0$ and $\ell \in\{1, \ldots, n\}$ with $h_{-, \ell}(\underline{\boldsymbol{\lambda}}) \leqslant m$. For such an $m$, we can always take $\ell=n$, since if $h_{-, n}(\underline{\boldsymbol{\lambda}}) \geqslant m$, then $F^{m} L(\underline{\boldsymbol{\lambda}}) \neq 0$. Indeed, $L\left({ }_{n} \tilde{f}^{m} \underline{\boldsymbol{\lambda}}\right)$ appears as in $F^{m} L(\underline{\boldsymbol{\lambda}})$, this is proved similarly to the checking $a_{i}=b_{i}$ in the proof of Theorem 6.1 . For each pair of labels $\underline{\boldsymbol{\lambda}}, \underline{\boldsymbol{\mu}}$, we can define

$$
\alpha_{\ell, m}(\underline{\boldsymbol{\lambda}}, \underline{\boldsymbol{\mu}})= \begin{cases}1 & h_{-}\left(\mu_{\ell}\right) \geqslant h_{-}\left(\lambda_{\ell}\right)=m \text { and } h_{-, \ell}(\underline{\boldsymbol{\mu}})>h_{-, \ell+1}(\underline{\boldsymbol{\mu}}) \\ 0 & \text { otherwise. }\end{cases}
$$

Proposition 7.4. Assume that $\tilde{f} L\left(\underline{\boldsymbol{\lambda}}^{\prime}\right)=L\left(\tilde{f} \underline{\boldsymbol{\lambda}}^{\prime}\right)$ holds whenever $F^{m-1} L\left(\underline{\boldsymbol{\lambda}}^{\prime}\right)=0$ and $\left|\varrho\left(\underline{\boldsymbol{\lambda}}^{\prime}\right)\right|-|\varrho(\underline{\boldsymbol{\lambda}})|$ is a multiple of $\alpha_{i}$. Then, in the above notation, we have

$$
\operatorname{Ext}^{r}(\Delta(\underline{\boldsymbol{\mu}}), L(\underline{\boldsymbol{\lambda}}))=0 \quad \text { for } \quad r \leqslant h_{-, \ell}(\underline{\boldsymbol{\mu}})-m-\alpha_{\ell, m}(\underline{\boldsymbol{\lambda}}, \underline{\boldsymbol{\mu}}) .
$$

Our proof closely follows that of [Losa, Proposition 5.5]. We will need an analog of [Losa, Lemma 5.6]. To state it, we need some more notation. Pick a label $\underline{\boldsymbol{\mu}}$. Let $\Lambda_{j}(\underline{\boldsymbol{\mu}})$ denote the set of labels in the labeling set $\Lambda$ for $\mathcal{C}$ consisting of all $\underline{\boldsymbol{\mu}}^{\prime}=\left(\mu_{1}^{\prime}, \ldots, \mu_{\ell}^{\prime}\right)$ such that the projective $P_{\mathcal{C}^{j}}\left(\mu_{j}^{\prime}\right)$ appears in ${ }_{j} E P_{\mathcal{C}^{j}}\left(\mu_{j}\right)$ and $\mu_{p}^{\prime}=\mu_{p}$ for $p \neq j$.

Lemma 7.5. Let a label $\mu$ and an integer $\ell$ be such that $h_{-, \ell}(\mu)>h_{-, \ell+1}(\mu)$. Set $\underline{\overline{\boldsymbol{\mu}}}:=\ell \underline{\tilde{f}} \underline{\boldsymbol{\mu}}$. Let $\mu^{\prime} \in \Lambda_{j}(\underline{\overline{\boldsymbol{\mu}}})$. Then the following holds: 
(1) If $j<\ell$, then $h_{-, \ell}\left(\underline{\mu}^{\prime}\right)=h_{-, \ell}(\overline{\boldsymbol{\mu}})=h_{-, \ell}(\boldsymbol{\mu})-1$.

(2) If $j=\ell$ and $\underline{\boldsymbol{\mu}}^{\prime} \neq \underline{\boldsymbol{\mu}}$, then $h_{-, \ell}\left(\underline{\boldsymbol{\mu}}^{\prime}\right)>h_{-, \ell}(\underline{\boldsymbol{\mu}})$.

(3) If $j>\ell$, then $h_{-, \ell}\left(\underline{\boldsymbol{\mu}}^{\prime}\right)>h_{-, \ell}(\underline{\boldsymbol{\mu}})$.

Proof. The first claim follows from $\underline{\boldsymbol{\mu}}_{p}^{\prime}=\underline{\boldsymbol{\mu}}_{p}$ for $p \geqslant \ell$. To prove the second claim we note that if $P_{\mathcal{C}^{j}}\left(\mu_{j}^{\prime}\right)$ appears in ${ }_{j} E P_{\mathcal{C}^{j}}\left(\mu_{j}\right)$, then $h_{-}\left(L_{\mathcal{C}^{j}}\left(\mu_{j}^{\prime}\right)\right) \geqslant h_{-}\left(L_{\mathcal{C}^{j}}\left(\mu_{j}\right)\right)+1$ with the equality if and only if $\mu_{j}^{\prime}={ }_{j} \tilde{e} \mu_{j}$. This follows from [CR08, Proposition 5.20] (applied to ${ }_{j} F$ using $\left.\operatorname{Hom}\left({ }_{j} E P_{\mathcal{C}^{j}}\left(\mu_{j}^{\prime}\right), L_{\mathcal{C}^{j}}(\xi)\right)=\operatorname{Hom}\left(P_{\mathcal{C}^{j}}\left(\mu_{j}^{\prime}\right),{ }_{j} F L_{\mathcal{C}^{j}}(\xi)\right)\right)$. Now the claims of $(2)$ and (3) become purely combinatorial and have proofs like those of [Losa, Lemma 5.6].

Proof of Proposition 7.4. We may assume that $h_{-, \ell}(\boldsymbol{\mu})>h_{-, \ell+1}(\underline{\boldsymbol{\mu}})$, since otherwise the desired equality is a special case of the same claim for $\ell+1$. This is equivalent to requiring that $\overline{\boldsymbol{\mu}}:=\ell \tilde{f} \boldsymbol{\mu}$ is not 0 .

It is a consequence of the hypotheses that given $m, h:=h_{-, \ell}(\boldsymbol{\mu})$ and $r \leqslant h-m$, the following claims hold:

(i) $\operatorname{Ext}^{r^{\prime}}\left(\Delta\left(\underline{\boldsymbol{\mu}}^{\prime}\right), L\left(\underline{\boldsymbol{\lambda}}^{\prime}\right)\right)=0$ provided $F^{m-1} L\left(\underline{\boldsymbol{\lambda}}^{\prime}\right)=0$ and $r^{\prime} \leqslant h_{-}\left(\underline{\boldsymbol{\mu}^{\prime}}\right)-m+1$ (in this case, by the assumption of the proposition, we have $\alpha_{m-1, \ell^{\prime}}=0$ for all $\left.\ell^{\prime}\right)$.

(ii) We have $\operatorname{Ext}^{r^{\prime}}\left(\Delta\left(\underline{\boldsymbol{\mu}}^{\prime}\right), L\left(\underline{\boldsymbol{\lambda}}^{\prime}\right)\right)=0$ for all $\underline{\boldsymbol{\lambda}}^{\prime}, \underline{\boldsymbol{\mu}}^{\prime}$ with $F^{m} L\left(\underline{\boldsymbol{\lambda}}^{\prime}\right)=0$ and all $r^{\prime} \leqslant$ $\min \left(h_{-, \ell}\left(\underline{\boldsymbol{\mu}}^{\prime}\right)-m-\alpha_{\ell, m}\left(\underline{\boldsymbol{\lambda}}^{\prime}, \underline{\boldsymbol{\mu}}^{\prime}\right), r-1\right)$.

(iii) We have $\operatorname{Ext}^{r^{\prime}}\left(\Delta\left(\underline{\boldsymbol{\mu}}^{\prime}\right), L\left(\underline{\boldsymbol{\lambda}}^{\prime}\right)\right)=0$ for all $\underline{\boldsymbol{\lambda}}^{\prime}$ such that $F^{m} L\left(\underline{\boldsymbol{\lambda}}^{\prime}\right)=0$, all $\underline{\boldsymbol{\mu}}$ such that $h_{-, \ell}(\underline{\boldsymbol{\mu}})>h$ and all $r^{\prime} \leqslant h_{-, \ell}\left(\underline{\boldsymbol{\mu}}^{\prime}\right)-m-\alpha_{m, \ell}\left(\underline{\boldsymbol{\lambda}}^{\prime}, \underline{\boldsymbol{\mu}}^{\prime}\right)$.

We can assume (i) because, thanks to the assumption of the Proposition, we can take $\ell=1$ as long as $F^{m-1} L\left(\underline{\boldsymbol{\lambda}}^{\prime}\right)=0$. We can assume (iii) because there are only finitely many $\boldsymbol{\mu}$ such that $\Delta(\underline{\boldsymbol{\mu}})$ and $L(\underline{\boldsymbol{\lambda}})$ lie in the same block (all weight subspaces in $V_{1} \otimes \ldots \otimes V_{n}$ are finite dimensional). Also we remark that we may assume that $r>0$ : the case $r=0$ follows from $\underline{\boldsymbol{\lambda}} \neq \underline{\boldsymbol{\mu}}$ (the latter holds because, whenever we consider $r=0$, we have $h_{-, \ell}(\underline{\boldsymbol{\lambda}})<m \leqslant h_{-, \ell}(\underline{\boldsymbol{\mu}})$ or $h_{-, \ell}(\underline{\boldsymbol{\lambda}})=m<h_{-, \ell}(\underline{\boldsymbol{\mu}})$, or $\left.h_{-}\left(\lambda_{\ell}\right)<h_{-}\left(\mu_{\ell}\right)\right)$.

Consider the object $\bar{E} \Delta(\underline{\bar{\mu}})$ and its filtration described in (TPC3). Let $\mathcal{F}$ denote the filtration subobject with successive filtration quotients $\Delta\left({ }_{j} E P_{\varrho(\underline{\bar{\mu}})}(\underline{\overline{\boldsymbol{\mu}}})\right), j \leqslant \ell$. The object $\mathcal{F}$ has quotient isomorphic to $h_{+}\left(\bar{\mu}_{\ell}\right)$ copies of $\Delta(\underline{\boldsymbol{\mu}})$. Let $\mathcal{F}_{0}$ denote the kernel of this quotient. Then all standards occurring in the filtration of $\mathcal{F}_{0}$ satisfy (1) or (2) of Lemma 7.5, while all standards in the filtration of $E \Delta(\overline{\boldsymbol{\mu}}) / \mathcal{F}$ satisfy (3) of Lemma 7.5. We also remark that $\alpha_{m, \ell}\left(\underline{\boldsymbol{\mu}^{\prime}}, \underline{\boldsymbol{\lambda}}\right) \leqslant \alpha_{m, \ell}(\underline{\boldsymbol{\mu}}, \underline{\boldsymbol{\lambda}})$ when $j \neq \ell$. Indeed, otherwise $h_{-}\left(\mu_{\ell}^{\prime}\right) \geqslant m$ and $h_{-}\left(\mu_{\ell}\right)<m$ that is impossible because $\mu_{\ell}^{\prime}=\bar{\mu}_{\ell}$ and $h_{-}\left(\bar{\mu}_{\ell}\right)=h_{-}\left(\mu_{\ell}\right)-1$. So, thanks to Lemma 7.5, for any subquotient $\Delta\left(\boldsymbol{\mu}^{\prime}\right)$ of $E \Delta(\bar{\mu}) / \mathcal{F}$ we have $h_{-, \ell}\left(\boldsymbol{\mu}^{\prime}\right)-m-\alpha_{m, \ell}\left(\boldsymbol{\mu}^{\prime}, \underline{\boldsymbol{\lambda}}\right) \geqslant r+1$, while for all subquotients $\Delta\left(\underline{\boldsymbol{\mu}}^{\prime}\right)$ of $\mathcal{F}_{0}$ the inequality $h_{-, \ell}\left(\underline{\boldsymbol{\mu}}^{\prime}\right)-m-\alpha_{m, \ell}\left(\underline{\boldsymbol{\mu}}^{\prime}, \underline{\boldsymbol{\lambda}}\right) \geqslant r-1$ holds.

It follows from (iii) that

$$
\operatorname{Ext}^{r+1}(E \Delta(\underline{\overline{\boldsymbol{\mu}}}) / \mathcal{F}, L(\underline{\boldsymbol{\lambda}}))=0 .
$$

It follows from (ii) that

$$
\operatorname{Ext}^{r-1}\left(\mathcal{F}_{0}, L(\underline{\boldsymbol{\lambda}})\right)=0 \text {. }
$$

We claim that

$$
\operatorname{Ext}^{r}(E \Delta(\underline{\bar{\mu}}), L(\underline{\boldsymbol{\lambda}}))=0 .
$$


Indeed, by the biadjointness, $\operatorname{Ext}^{r}(E \Delta(\underline{\bar{\mu}}), L(\underline{\boldsymbol{\lambda}}))=\operatorname{Ext}^{r}(\Delta(\underline{\bar{\mu}}), F L(\underline{\boldsymbol{\lambda}}))$. All simple constituents $L\left(\underline{\boldsymbol{\lambda}}^{\prime}\right)$ of $F L(\underline{\boldsymbol{\lambda}})$ satisfy the assumptions of (i). Since $h_{-, \ell}(\underline{\boldsymbol{\mu}})=h-1$ and $r \leqslant$ $(h-1)-(m-1)=h-m$, we deduce (7.3) from (i).

Using standard short exact sequences for Ext's (compare with the proof of [Losa, Proposition 5.5]) together with $(\underline{7.1}),(17.2),(17.3)$, we see that $\operatorname{Ext}^{r}\left(\mathcal{F} / \mathcal{F}_{0}, L(\underline{\boldsymbol{\lambda}})\right)=0$. Since $\mathcal{F} / \mathcal{F}_{0}$ is the direct sum of several copies of $\Delta(\underline{\boldsymbol{\mu}})$, we are done.

7.3. Proof of the main theorem. Our proof basically repeats that in [Losa]. Fix a weight $\mu$ for $\mathfrak{g}$. Of course, we can restrict our attention to the weight subcategories with weights of the form $\mu+r \alpha_{i}, r \in \mathbb{Z}$.

Now, let us prove the claim that our crystal operators agree by induction on $w(\underline{\boldsymbol{\lambda}})=$ $\left\langle\varrho(\underline{\boldsymbol{\lambda}}), \alpha_{i}^{\vee}\right\rangle$. The set of values of $w(\underline{\boldsymbol{\lambda}})$ is finite since our representations are integrable. In order to organize our induction, we consider two statements:

$\left(\phi_{w}\right)$ For all $L(\underline{\boldsymbol{\lambda}})$ with $w(\underline{\boldsymbol{\lambda}}) \leqslant w$, we have that $\tilde{f} L(\underline{\boldsymbol{\lambda}})=L(\tilde{f} \underline{\boldsymbol{\lambda}})$.

$\left(\eta_{w}\right)$ We have both $\left(\phi_{w-1}\right)$ and that $h_{-}(\underline{\boldsymbol{\lambda}})=h_{-}(L(\underline{\boldsymbol{\lambda}}))$ for all $\underline{\boldsymbol{\lambda}}$ with $w(\underline{\boldsymbol{\lambda}}) \leqslant w$.

Obviously, proving $\left(\phi_{w}\right)$ for all $w$ will complete the proof of Theorem 7.2 , we will proceed in establishing $\left(\phi_{w-1}\right) \Rightarrow\left(\eta_{w}\right) \Rightarrow\left(\phi_{w}\right)$.

The base of induction is the statement $\left(\phi_{w}\right)$ where we take $w$ to be the minimal amongst those realized. In this case, both sides of the equality are 0.

Proof that $\left(\phi_{w-1}\right) \Rightarrow\left(\eta_{w}\right)$. First, we establish the inequality $h_{-}(L(\underline{\boldsymbol{\lambda}})) \geqslant h_{-}(\underline{\boldsymbol{\lambda}})$. Assume the contrary, $h_{-}(L(\underline{\boldsymbol{\lambda}}))<h_{-}(\underline{\boldsymbol{\lambda}})$. Then $h_{-}(\underline{\boldsymbol{\lambda}})>0$ and so the element $\underline{\overline{\boldsymbol{\lambda}}}:=\tilde{f} \underline{\boldsymbol{\lambda}}$ is nonzero. Let $j \in\{1, \ldots, n\}$ be the unique index such that $\underline{\bar{\lambda}}={ }_{j} \tilde{f} \underline{\boldsymbol{\lambda}}$. Consider the object $E \Delta(\underline{\bar{\lambda}})$. Form the filtered subobjects $\mathcal{F}_{0}, \mathcal{F}$ as in the proof of Proposition 7.4 so that $\mathcal{F} / \mathcal{F}_{0}$ is the direct sum of several copies of $\Delta(\underline{\boldsymbol{\lambda}})$.

Pick $\Delta(\underline{\boldsymbol{\mu}})$ appearing in $E \Delta(\underline{\overline{\boldsymbol{\lambda}}}) / \mathcal{F}$ and set $m=h_{-}(\underline{\boldsymbol{\lambda}}), \ell=j$ so that $h_{-}(\underline{\boldsymbol{\lambda}})=h_{-, \ell}(\underline{\boldsymbol{\lambda}})$. Since $h_{-}\left(\overline{\mu_{j}}\right)=h_{-}\left(\lambda_{j}\right)-1$, we have $\alpha_{m, \ell}(\underline{\boldsymbol{\mu}}, \underline{\boldsymbol{\lambda}})=0$. Also by (3) of Lemma 7.5, $h_{-, \ell}(\underline{\boldsymbol{\mu}})>m$. The assumptions of Proposition 7.4 are satisfied. It follows that $\operatorname{Ext}^{1}(\Delta(\underline{\boldsymbol{\mu}}), L(\underline{\boldsymbol{\lambda}}))=0$. Thus, $L(\underline{\boldsymbol{\lambda}})$ lies in the head of $E \Delta(\underline{\overline{\boldsymbol{\lambda}}})$.

By [CR08, Lemma 5.11], this implies that $h_{-}(L(\underline{\boldsymbol{\lambda}})) \geqslant h_{-}(L(\underline{\overline{\boldsymbol{\lambda}}}))+1$. By the inductive assumption,

$$
h_{-}(L(\underline{\overline{\boldsymbol{\lambda}}}))=h_{-}(\underline{\overline{\boldsymbol{\lambda}}})=h_{-}(\underline{\boldsymbol{\lambda}})-1 \text {. }
$$

So we see that $h_{-}(L(\underline{\boldsymbol{\lambda}})) \geqslant h_{-}(\underline{\boldsymbol{\lambda}})$, our desired inequality in the weight subcategories with $w(\underline{\boldsymbol{\lambda}})=w$.

Note that the crystal of $\mathcal{C}$ is isomorphic to the crystal of $V_{1} \otimes V_{2} \otimes \ldots \otimes V_{n}$ by [BK07, 5.55] combined with [CR08, Proposition 5.20]. Our crystal on $\Lambda$ is also isomorphic to that of $V_{1} \otimes V_{2} \otimes \ldots \otimes V_{n}$, since it is the standard tensor product crystal structure. In particular, we see that for any $w, h$ the number of $\underline{\boldsymbol{\lambda}}$ with $w(\underline{\boldsymbol{\lambda}})=w$ and $h_{-}(L(\underline{\boldsymbol{\lambda}}))=h$ equals to the number of $\underline{\boldsymbol{\lambda}}$ with $w(\underline{\boldsymbol{\lambda}})=w$ and $h_{-}(\underline{\boldsymbol{\lambda}})=h$. So the inequality $h_{-}(L(\underline{\boldsymbol{\lambda}})) \geqslant h_{-}(\underline{\boldsymbol{\lambda}})$ must be an equality for all $\underline{\boldsymbol{\lambda}}$ with $w(\underline{\boldsymbol{\lambda}})=w$; that is, the implication $\left(\phi_{w-1}\right) \Rightarrow\left(\eta_{w}\right)$ is now proved.

Proof that $\left(\eta_{w}\right) \Rightarrow\left(\phi_{w}\right)$. It suffices to prove instead that that $\tilde{e} L\left(\underline{\boldsymbol{\lambda}}^{\prime}\right)=L\left(\tilde{e} \underline{\boldsymbol{\lambda}}^{\prime}\right)$ for all $\underline{\boldsymbol{\lambda}}^{\prime}$ with $w\left(\underline{\boldsymbol{\lambda}}^{\prime}\right)=w-2$.

First of all, we have that $\tilde{e} L(\underline{\boldsymbol{\lambda}})=0$ if and only if $\tilde{e} \underline{\boldsymbol{\lambda}}=0$. Indeed, since $h_{-}(L(\underline{\boldsymbol{\lambda}}))=h_{-}(\underline{\boldsymbol{\lambda}})$, we know that $h_{+}(L(\underline{\boldsymbol{\lambda}}))=h_{+}(\underline{\boldsymbol{\lambda}})$. So we may assume that $\tilde{e} L(\underline{\boldsymbol{\lambda}}) \neq 0 \neq \tilde{e} \underline{\boldsymbol{\lambda}}$. 
We say that $\underline{\boldsymbol{\lambda}}$ and $\underline{\boldsymbol{\lambda}}^{\prime}$ lie in the same $i$-family if one can obtain $\underline{\boldsymbol{\lambda}}$ from $\underline{\boldsymbol{\lambda}}^{\prime}$ by applying maps ${ }_{j} \tilde{e}_{i}$ and ${ }_{j} \tilde{f}_{i}$. We will order elements of the family using reverse lexicographic (i.e. reading from the right) order $\succ$ on the $i$-signatures with $+>-$. In our proof we may assume that $\tilde{e} L\left(\underline{\boldsymbol{\lambda}}^{\prime}\right)=L\left(\tilde{e} \underline{\boldsymbol{\lambda}}^{\prime}\right)$ is proved for all $\underline{\boldsymbol{\lambda}}^{\prime}$ such that $h_{-}\left(\underline{\boldsymbol{\lambda}}^{\prime}\right)=h_{-}(\underline{\boldsymbol{\lambda}})$ and $\tilde{e} \underline{\boldsymbol{\lambda}} \succ \tilde{e} \underline{\boldsymbol{\lambda}}^{\prime}$.

Now, fix $\underline{\boldsymbol{\lambda}}$ and define $\underline{\tilde{\boldsymbol{\lambda}}}$ by $\tilde{e} L(\underline{\boldsymbol{\lambda}})=L(\underline{\tilde{\boldsymbol{\lambda}}})$. We already know that

$$
h_{-}(\tilde{e} \underline{\boldsymbol{\lambda}})=h_{-}(\underline{\tilde{\boldsymbol{\lambda}}})=h_{-}(\underline{\boldsymbol{\lambda}})+1 \text {. }
$$

By Lemma 7.3, we know that $\tilde{e} \underline{\boldsymbol{\lambda}}$ is amongst the labels ${ }_{j} \tilde{e} \underline{\boldsymbol{\lambda}}$ with $h_{-}\left({ }_{j} \tilde{e} \underline{\boldsymbol{\lambda}}\right)=h_{-}(\underline{\boldsymbol{\lambda}})+1$.

Assume that $\underline{\tilde{\boldsymbol{\lambda}}} \neq \tilde{e} \underline{\boldsymbol{\lambda}}$. Since $h_{-}(\underline{\tilde{\boldsymbol{\lambda}}})-1=h_{-}(\underline{\boldsymbol{\lambda}}) \geqslant 0$, we see that $\underline{\boldsymbol{\lambda}}^{\prime}:=\tilde{f} \tilde{\boldsymbol{\lambda}} \neq 0$ and so $\underline{\tilde{\boldsymbol{\lambda}}}=\tilde{e} \underline{\boldsymbol{\lambda}}^{\prime}$. If $\tilde{e} \underline{\boldsymbol{\lambda}} \succ \underline{\tilde{\boldsymbol{\lambda}}}=\tilde{e} \underline{\boldsymbol{\lambda}}^{\prime}$, then by the inductive assumption

$$
\tilde{e} L\left(\underline{\boldsymbol{\lambda}}^{\prime}\right)=L\left(\tilde{e} \underline{\boldsymbol{\lambda}}^{\prime}\right)=L(\underline{\tilde{\boldsymbol{\lambda}}})=\tilde{e} L(\underline{\boldsymbol{\lambda}})
$$

which is impossible.

Thus, we must have $\underline{\tilde{\boldsymbol{\lambda}}} \succ \tilde{e} \underline{\boldsymbol{\lambda}}$. But then Lemma 7.5 implies $h_{-}(\underline{\tilde{\boldsymbol{\lambda}}})>h_{-}(\tilde{e} \underline{\boldsymbol{\lambda}})$, which is another contradiction. This proves $\tilde{e} L(\underline{\boldsymbol{\lambda}})=L(\tilde{e} \underline{\boldsymbol{\lambda}})$, and thus implies $\left(\phi_{w}\right)$. This closes the circle of the induction and completes the proof.

\section{REFERENCES}

[BK07] Arkady Berenstein and David Kazhdan, Geometric and unipotent crystals. II. From unipotent bicrystals to crystal bases, Quantum groups, Contemp. Math., vol. 433, Amer. Math. Soc., Providence, RI, 2007, pp. 13-88.

[BLW] Jon Brundan, Ivan Losev, and Ben Webster, Graded tensor product categorifications and the super Kazhdan-Lusztig conjecture, in preparation.

[CL] Sabin Cautis and Aaron Lauda, Implicit structure in 2-representations of quantum groups, arXiv:1111.1431.

[CPS96] Edward Cline, Brian Parshall, and Leonard Scott, Stratifying endomorphism algebras, Mem. Amer. Math. Soc. 124 (1996), no. 591, viii+119.

[CR08] Joseph Chuang and Raphaël Rouquier, Derived equivalences for symmetric groups and $\mathfrak{s}_{2}$ categorification, Ann. of Math. (2) 167 (2008), no. 1, 245-298.

[HY] Jiuzu Hong and Oded Yacobi, Polynomial representations of general linear groups and categorifications of Fock space, arXiv:1101.2456.

[KL09] Mikhail Khovanov and Aaron D. Lauda, A diagrammatic approach to categorification of quantum groups. I, Represent. Theory 13 (2009), 309-347. MR 2525917 (2010i:17023)

[KL10] - A categorification of quantum $\operatorname{sl}(n)$, Quantum Topol. 1 (2010), no. 1, 1-92. MR 2628852 (2011g:17028)

[Lau10] Aaron D. Lauda, A categorification of quantum sl(2), Adv. Math. 225 (2010), no. 6, 3327-3424. MR 2729010 (2012b:17036)

[Losa] Ivan Losev, Highest weight $\mathfrak{s l}_{2}$-categorifications I: crystals, arXiv:1201.4493,

[Losb] - Highest weight $\mathfrak{s l}_{2}$-categorifications II: structure theory, arXiv: 1203.5545,

[Losc] , Towards multiplicities for cyclotomic rational Cherednik algebras, arXiv:1207.1299.

[LV11] Aaron D. Lauda and Monica Vazirani, Crystals from categorified quantum groups, Adv. Math. 228 (2011), no. 2, 803-861. MR 2822211

[Rou] Raphael Rouquier, 2-Kac-Moody algebras, arXiv:0812.5023.

[Rou08] Raphäl Rouquier, q-Schur algebras and complex reflection groups, Mosc. Math. J. 8 (2008), no. 1, 119-158, 184. MR 2422270 (2010b:20081)

[Sha11] Peng Shan, Crystals of Fock spaces and cyclotomic rational double affine Hecke algebras, Ann. Sci. Éc. Norm. Supér. (4) 44 (2011), no. 1, 147-182. MR 2760196 (2012c:20009)

[Soe90] Wolfgang Soergel, Kategorie $\mathcal{O}$, perverse Garben und Moduln über den Koinvarianten zur Weylgruppe, J. Amer. Math. Soc. 3 (1990), no. 2, 421-445. MR MR1029692 (91e:17007)

[Weba] Ben Webster, Knot invariants and higher representation theory I: diagrammatic and geometric categorification of tensor products, arXiv:1001.2020, 
[Webb] _ Knot invariants and higher representation theory II: categorification of quantum knot invariants, arXiv:1005.4559.

Department of Mathematics, Northeastern University, Boston MA 02115 USA

E-mail address: i.loseu@neu.edu; b.webster@neu.edu 